\title{
Reperfusion Therapy with Rapamycin Attenuates Myocardial Infarction through Activation of AKT and ERK
}

\author{
Scott M. Filippone, Arun Samidurai, Sean K. Roh, Chad K. Cain, Jun He, \\ Fadi N. Salloum, Rakesh C. Kukreja, and Anindita Das \\ Pauley Heart Center, Department of Internal Medicine, Division of Cardiology, Virginia Commonwealth University, \\ Richmond, VA 23298, USA \\ Correspondence should be addressed to Anindita Das; anindita.das@vcuhealth.org
}

Received 2 December 2016; Revised 26 January 2017; Accepted 14 February 2017; Published 8 March 2017

Academic Editor: Massimo Collino

Copyright (C) 2017 Scott M. Filippone et al. This is an open access article distributed under the Creative Commons Attribution License, which permits unrestricted use, distribution, and reproduction in any medium, provided the original work is properly cited.

Prompt coronary reperfusion is the gold standard for minimizing injury following acute myocardial infarction. Rapamycin, mammalian target of Rapamycin (mTOR) inhibitor, exerts preconditioning-like cardioprotective effects against ischemia/reperfusion (I/R) injury. We hypothesized that Rapamycin, given at the onset of reperfusion, reduces myocardial infarct size through modulation of mTOR complexes. Adult C57 male mice were subjected to 30 min of myocardial ischemia followed by reperfusion for 1 hour/24 hours. Rapamycin $(0.25 \mathrm{mg} / \mathrm{kg})$ or DMSO $(7.5 \%)$ was injected intracardially at the onset of reperfusion. Post-I/R survival (87\%) and cardiac function (fractional shortening, FS: $28.63 \pm 3.01 \%$ ) were improved in Rapamycin-treated mice compared to DMSO (survival: $63 \%$, FS: $17.4 \pm 2.6 \%$ ). Rapamycin caused significant reduction in myocardial infarct size (IS: $26.2 \pm 2.2 \%)$ and apoptosis $(2.87 \pm 0.64 \%)$ as compared to DMSO-treated mice (IS: $47.0 \pm 2.3 \%$; apoptosis: $7.39 \pm 0.81 \%$ ). Rapamycin induced phosphorylation of AKT S473 (target of mTORC2) but abolished ribosomal protein S6 phosphorylation (target of mTORC1) after I/R. Rapamycin induced phosphorylation of ERK1/2 but inhibited p38 phosphorylation. Infarct-limiting effect of Rapamycin was abolished with ERK inhibitor, PD98059. Rapamycin also attenuated Bax and increased Bcl-2/Bax ratio. These results suggest that reperfusion therapy with Rapamycin protects the heart against I/R injury by selective activation of mTORC2 and ERK with concurrent inhibition of mTORC1 and p38.

\section{Introduction}

Timely intervention at reperfusion is the current standard of care for the treatment of an acute myocardial infarction (AMI). However, the consequences of reperfusion injury still represent a major limitation to treatment. Therefore, developing novel approaches to minimize such injury is needed to further improve outcomes following an AMI.

The mammalian target of Rapamycin (mTOR) is a serine/threonine kinase that functions as a key regulator of cell growth, survival, and metabolism [1]. Identified as an intracellular sensor, mTOR detects nutrient and energy availability, as well as stressors affecting the cell [1]. Complex downstream signaling networks provide mTOR the ability to regulate autophagy, protein synthesis, cell polarity, and cytoskeletal organization [2-6]. For this reason, mTOR signaling has been studied extensively to elucidate its role in diabetes, aging, cancer, metabolic disorders, and cardiovascular diseases [2,7-12]. mTOR is also an integral component for cardiovascular development and its targeted deletion in the heart has been shown to result in cardiomyopathy, heart failure, and death [5].

Effective metabolic control is established via the formation of two distinct mTOR multiprotein complexes: mTOR complex 1 (mTORC1) and mTOR complex 2 (mTORC2) [6, $7,13]$. These complexes are responsible for the regulation of different cellular processes and are defined by the adaptor proteins that bind to the central mTOR catalytic subunit. Common to both complexes are DEPTOR (DEP domain containing mTOR-interacting protein), mLST8 (mammalian lethal with sec-13 protein 9), and a Ttil-Tel2 complex [6$8,10,12]$. Unique to $\mathrm{mTORC1}$ are Raptor (regulatoryassociated protein of mTOR) and the inhibitory PRAS40 (proline-rich AKT substrate) subunit which enable it to 
phosphorylate the downstream ribosomal protein $\mathrm{p} 70 \mathrm{~S} 6 \mathrm{~K}$ $\left(\mathrm{Ser}^{235 / 236}\right)$ and 4FBP1 (eukaryotic initiation factor 4E-binding protein 1) [14]. Activation of $\mathrm{mTORC1}$ is responsible for promoting autophagy and ribosome biogenesis $[12,15,16]$. mTORC2 contains Rictor (Rapamycin-insensitive companion of mTOR) and mSinl (mammalian stress-activated MAP kinase-interacting protein), allowing for phosphorylation of downstream $\mathrm{AKT}^{473}$ and GSK3ß [17]. Less is known about mTORC2 than its counterpart, but it has been proven to play a key role in cytoskeletal organization and cell growth/survival [18].

In 2006, our laboratory first identified the preconditioning-like cardioprotective effects of Rapamycin against ischemia/reperfusion (I/R) injury [9]. Rapamycin pretreatment reduced infarct size after $\mathrm{I} / \mathrm{R}$ injury and also attenuated necrosis and apoptosis in cardiomyocytes following simulated ischemia/reoxygenation (SI-RO). Mechanisms mediating the protective effects of Rapamycin involved the opening of mitochondrial ATP-sensitive potassium channels [9]. We further demonstrated that additional cardioprotective signaling occurs via phosphorylation of ERK, STAT3, and endothelial nitric oxide synthase (eNOS), in conjunction with an increased Bcl-2: Bax ratio [19]. Whether Rapamycin treatment attenuates reperfusion injury remains unknown. Therefore, the present study was designed to investigate the in vivo effects of reperfusion therapy with Rapamycin. Considering the important role of the Reperfusion Injury Salvage Kinase (RISK) pathway, a group of prosurvival protein kinases (including AKT and Erk1/2), which confer cardioprotection when activated specifically at the time of myocardial reperfusion [20, 21], we hypothesized that the RISK pathway may provide an amenable pharmacological target for cardioprotection with Rapamycin. Our results demonstrate that Rapamycin administered at the onset of reperfusion shows great promise as an interventional drug capable of significantly reducing infarct size and cardiomyocyte apoptosis following I/R injury via signaling pathways involving MAP kinases and the PI3K-AKT.

\section{Methods}

2.1. Chemicals and Reagents. Rapamycin (Sirolimus ${ }^{\circledR}$ ) was purchased from LC Laboratories (MA). PD98059, an ERK inhibitor, was purchased from Sigma Aldrich (St. Louis, MO). ApoAlert $^{\mathrm{TM}}$ DNA Fragmentation Assay kit was purchased from BD Biosciences, Palo Alto, CA. DAPI was bought from Vector Laboratories, Inc. CA. Antibodies for phosphoserine ${ }^{473}$ AKT, AKT, p-S6, S6, p-ERK1/2, ERK1/2, p-P38, P38, phospho-tyrosine ${ }^{705}$-STAT3, STAT3, Bcl-2, and Bax were purchased from Cell Signaling Technology. SOD-2, ferritin heavy chain, and GAPDH-HRP were purchased from Santa Cruz Biotechnology.

2.2. Animals. Adult C57BL/6J male mice (body weight $\sim 30 \mathrm{~g}$ ) were purchased from Jackson Laboratories. Animal care and experiments were approved by the Institutional Care and Use Committee of Virginia Commonwealth University and were conducted according to the Guide for the Care and Use of

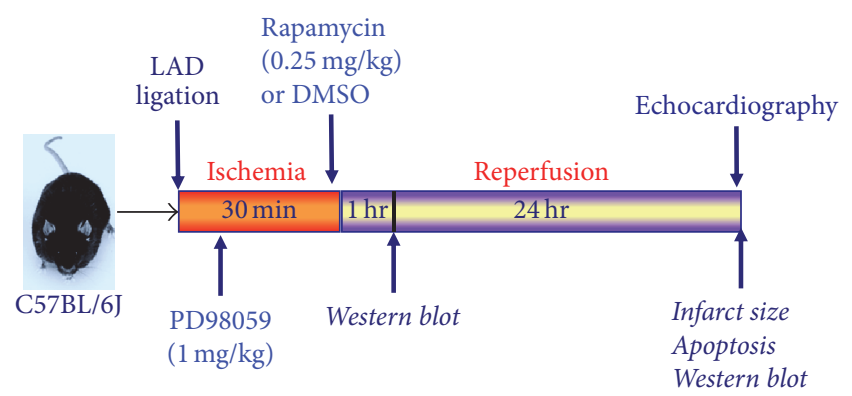

FIGURE 1: Experimental protocol. Experimental groups and protocol of regional I/R by LAD occlusion in C57 mouse hearts.

Laboratory Animals for Biomedical Research published by the National Institutes of Health (No. 85-23, revised 1996).

2.3. Experimental Groups. For in vivo regional I/R protocol, four groups were used: (1) DMSO (7.5\%), (2) Rapamycin $(0.25 \mathrm{mg} / \mathrm{kg})$, (3) Rapamycin + PD98059 (1 mg/kg an ERK inhibitor), and (4) PD98059 alone. DMSO or Rapamycin was injected (intracardiac, i.c.) 5 min before the onset of reperfusion following $30 \mathrm{~min}$ of in situ ischemia. ERK activation was inhibited through intraperitoneal administration of PD98059 10 minutes following the start of ischemia. The surgeon was blinded to the treatment allocation.

2.4. Myocardial Infarction Protocol. The in vivo myocardial $\mathrm{I} / \mathrm{R}$ procedures were conducted in mouse by ligation of the left anterior descending coronary artery (LAD) according to a previously reported method [22]. Animals were anesthetized with pentobarbital sodium $(70 \mathrm{mg} / \mathrm{kg}$, i.p. $)$ and ventilated on a positive pressure ventilator. A left thoracotomy was performed at the fourth intercostal space, and the heart was exposed by stripping the pericardium. To induce ischemia, the LAD was occluded for $30 \mathrm{~min}$ by a 7.0 silk ligature that was placed around it and a small piece of polyethylene tubing (PE10) that was positioned on top of it. Reperfusion was established by removing the PE10 tube that was compressing the LAD. 5 min prior to reperfusion, Rapamycin $(0.25 \mathrm{mg} / \mathrm{kg})$ or DMSO (7.5\%) was injected intracardially to ensure its immediate availability at the onset of reperfusion as well as avoiding any preconditioning effect. Although intracardial injection of Rapamycin is not clinically applicable, it resembles the best option to mimic intracoronary delivery during cardiac catheterization, which is not feasible in mice. We chose this route of administration, which has been previously reported in the literature with same myocardial ischemia/reperfusion protocol [23], to bypass liver metabolism that occurs with intravenous/intraperitoneal administration in order to ensure immediate availability of Rapamycin at the onset of reperfusion in our study. The dose of Rapamycin was chosen based on our previous studies on Rapamycin-induced cardioprotection against myocardial $I / R$ injury $[9,19]$. Air was then expelled from the chest, and the chest cavity was closed. The animal was placed in a cage on a heating pad until fully conscious and then kept for 24 hours (Figure 1). 
2.5. Measurement of Infarct Size. The heart was removed following $30 \mathrm{~min}$ of ischemia and 24 hours of reperfusion and mounted on a Langendorff apparatus. The coronary arteries were perfused with $37^{\circ} \mathrm{C}$ Krebs-Henseleit buffer. After the blood was washed out, $3 \mathrm{~mL}$ of $10 \%$ TTC in isotonic phosphate buffer ( $\mathrm{pH} \mathrm{7.4)}$ at $37^{\circ} \mathrm{C}$ was infused over several minutes before the ligature was retightened and $\sim 1 \mathrm{~mL}$ of $5 \%$ Phthalo blue dye was injected as a bolus into the aorta until most of the heart turned blue. The heart was perfused with saline to wash out the excess dye. After freezing the heart for 24 hours, it was sectioned from apex to base into slices of equal thickness $(\sim 1 \mathrm{~mm})$. The slices were then fixed in $10 \%$ neutral buffered formaldehyde for 4 hours with a weight on top to keep the heart slices flat for the initial $30 \mathrm{~min}$. The areas of infarcted tissue, the risk zone, and the whole left ventricle were determined by computer morphometry using ImageJ imaging software (NIH, Bethesda).

2.6. Echocardiography. Cardiac function was assessed via echocardiography using a VisualSonics Vevo 2100 Imaging System. Mice were anesthetized with $2.5 \mathrm{~L} / \mathrm{min}$ Isoflurane prior to assessment. Imaging was conducted through the parasternal short axis while mice were exposed to $1.5 \mathrm{~L} / \mathrm{min}$ Isoflurane via a nose cone. Left ventricular (LV) end-diastolic diameter (LVEDD) and end-systolic diameter (LVESD), LV fractional shortening (FS), and ejection fraction (EF) were calculated using Vevo analysis software (version 2.2.3).

2.7. Apoptosis Assay. Apoptosis was determined by TUNEL staining using an ApoAlert ${ }^{\mathrm{TM}}$ DNA Fragmentation Assay kit (BD Biosciences, CA) according to the manufacturer's protocol. Hearts were stored in a $10 \%$ formalin solution, and paraffin embedded tissue section was mounted on glass slides. Apoptosis was then assessed in the transverse sections of paraffin sections as previously reported [19]. Apoptotic cells were examined under a fluorescence microscope (Nikon Eclipse $\mathrm{T} i$ ) which were clearly identified with a strong nuclear green fluorescence. All cell nuclei were visualized as blue fluorescence following staining with DAPI. The apoptotic index was expressed as the number of apoptotic cells of all cardiomyocytes per field. Apoptotic rate in the peri-infarct regions was calculated using 6 random fields.

Lipid Peroxidation Assay. Lipid peroxidation in heart was assayed by measuring malondialdehyde (MDA) using a lipid peroxidation assay kit (BioVision, CA, USA) according to the manufacturer's protocol as previously reported [7].

2.8. Western Blots. Total soluble protein was extracted from the whole heart tissues (following $30 \mathrm{~min}$ ischemia and $1 \mathrm{hr}$ reperfusion or $24 \mathrm{hr}$ reperfusion) with extraction buffer. Seventy-five $(75 \mu \mathrm{g})$ proteins from each sample were separated by SDS-PAGE and transferred onto nitrocellulose membrane ( $0.2 \mathrm{um}$ pore size). The membrane was incubated in cold conditions overnight with primary antibodies

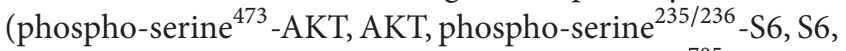
p-ERK1/2, ERK1/2, p-P38, P38, phospho-tyrosin ${ }^{705}$-STAT3, STAT3, Bcl-2, Bax, SOD-2, and ferritin heavy chain 1 or
GAPDH-HRP) in a 1:1000 dilution with 5\% BSA. The membrane was washed and incubated with horseradish peroxidase conjugated secondary antibody (1:3000 dilution in 5\% milk solution) and the blots were developed using a chemiluminescent system (Western Lighting Plus-ECL; Perkin Elmer, Inc.).

2.9. Data Analysis and Statistics. Statistical analysis was performed with GraphPad Prism 7 (Graphpad Software Inc.). Data are presented as mean \pm SEM. The differences between groups were analyzed with one-way analysis of variance (ANOVA) followed by Student-Newman-Keuls post hoc test for pairwise comparison, or by unpaired $t$-test. $p<0.05$ was considered to be statistically significant.

\section{Results}

3.1. Survival. A total of 105 mice were used in this study; 26 out of 30 mice survived in the Rapamycin-treated group (approximately 86\%) as compared with 27 out of 39 in the DMSO-treated group (69\%) following I/R. Post-I/R survival rate was $63 \%$ (14 out of 22 ) in mice treated with Rapamycin plus PD98059 and 57\% (8 out of 14) in mice treated with PD98059 alone.

3.2. Reperfusion Therapy with Rapamycin Reduces Infarct Size and Improves Cardiac Function. Rapamycin treatment at the onset of reperfusion resulted in a significant reduction in infarct size (\% of risk area) to $26.2 \pm 2.2 \%$ as compared to $47.0 \pm 2.3 \%$ in the DMSO-treated Control group $(n=6$, $p<0.0001$ ) after $30 \mathrm{~min}$ ischemia and 24-hour reperfusion (Figure 2(a)). Total risk area was not statistically different between Rapamycin $(55.5 \% \pm 2.5)$ and DMSO $(56.9 \% \pm 2.6)$ treated groups $(n=6, p>0.05)$ following in vivo $\mathrm{I} / \mathrm{R}$ (Figure 2(b)).

Cardiac function was assessed by echocardiography following $30 \mathrm{~min}$ ischemia by LAD occlusion and 24-hour reperfusion. DMSO-treated mice showed significant reduction of FS, $17.4 \pm 2.6 \%$, and EF, $35.2 \pm 4.99 \%$, after I/R as compared to Control mice before I/R (FS, $46.6 \pm 0.7 \%$, and EF, $81.0 \pm 2.1 \%)\left(n=6,{ }^{*} p<0.001\right.$ versus Control; Figures $2(\mathrm{c})$ and $2(\mathrm{~d}))$. Rapamycin improved post-I/R FS (28.63 $\pm 3.01 \%)$ and $\mathrm{EF}(52.22 \pm 4.1 \%)$. Heart rates did not change between the groups (Figure 2(e)).

3.3. Effect of Rapamycin on Myocardial Apoptosis. Following in vivo $\mathrm{I} / \mathrm{R}$, tissue sections from Rapamycin-treated hearts showed a significant reduction in TUNEL-positive nuclei in the peri-infarct regions $(2.87 \pm 0.64 \%)$ as compared to the DMSO-treated group $(7.39 \pm 0.81 \%, n=7, p<0.001)$ (Figures 3(a) and 3(b)).

3.4. Rapamycin Blocks Proapoptotic Bax Signaling. The expressions of Bcl-2 and Bax were measured to identify prosurvival and proapoptotic cell signaling, respectively. Bax expression was significantly increased at 24 hours following in vivo $\mathrm{I} / \mathrm{R}(n=5, p<0.0001)$ but was reduced to a preischemic state with administration of Rapamycin at reperfusion $(n=5$, $p<0.0001$ ) (Figures 3(c) and 3(d)). The expression of Bcl-2 


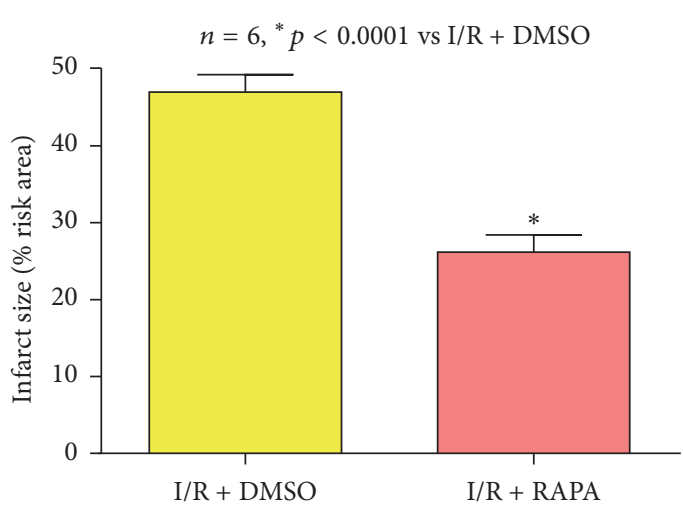

(a)

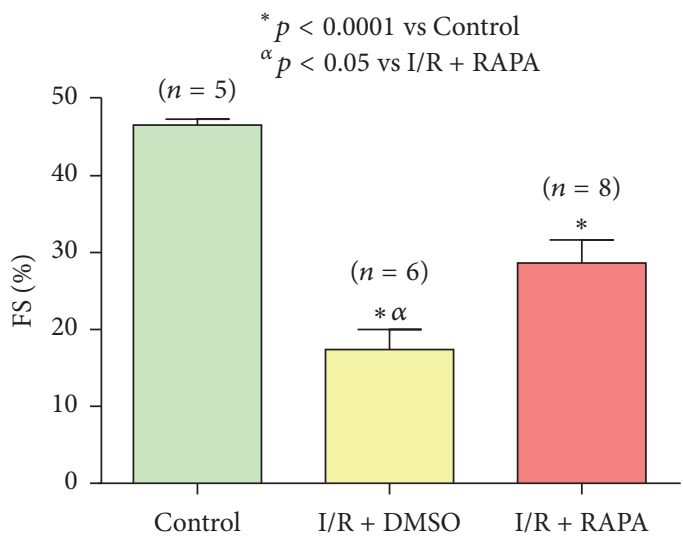

(c)

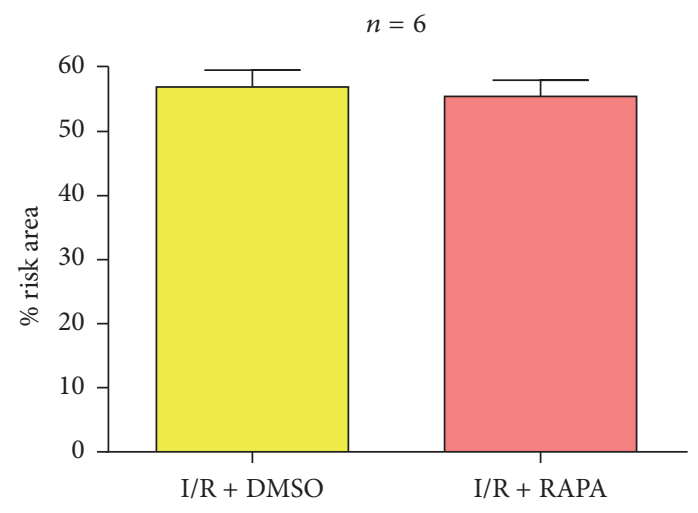

(b)

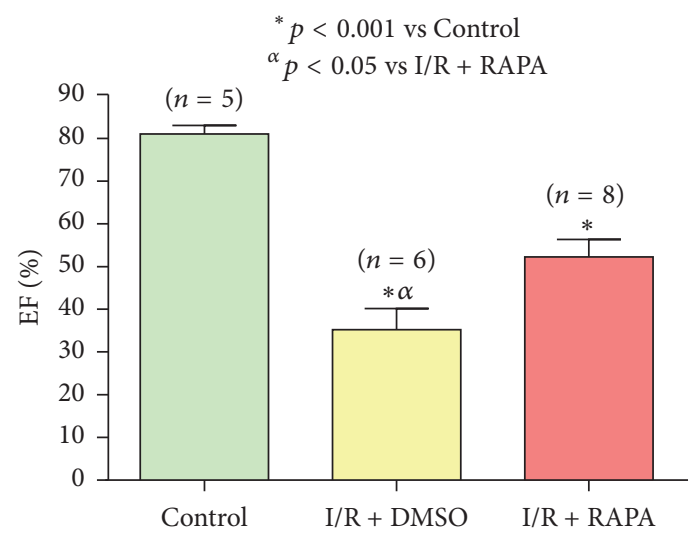

(d)

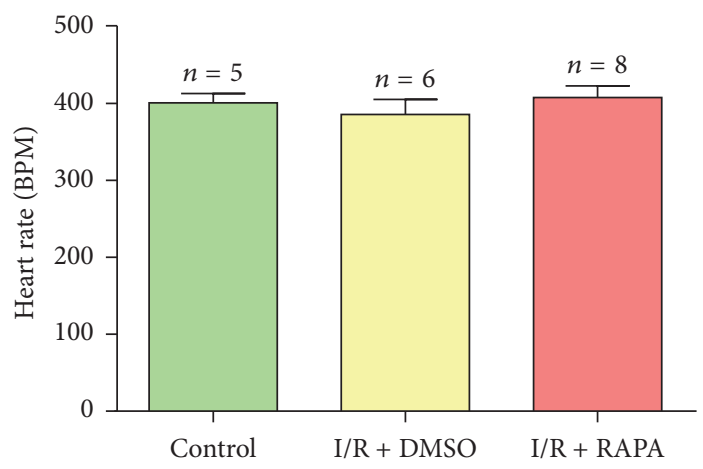

(e)

Figure 2: Rapamycin at the onset of reperfusion reduces infarct size. (a) Infarct size of C57 mouse hearts following 30 minutes of ischemia and 24 hours of reperfusion with and without Rapamycin treatment as percentage of risk area. After TTC and Phthalo blue staining, the blue area represents the remote area, the area at risk is stained red, and the infarcted myocardium is stained white. (b) Risk area as percentage of the overall tissue section. (c) Fractional shortening (\%) as measured via echocardiography. (d) Ejection fraction (\%). (e) Heart rate (BPM).

did not change following I/R injury with/without Rapamycin treatment (Figures 3(c) and 3(e)). However, the ratio of Bcl2/Bax significantly declined following I/R $(n=5, p<0.01)$ and was restored by Rapamycin treatment (Figure 3(f)).

3.5. Rapamycin Inhibits mTORC1 and Promotes mTORC2 Activity. To further elucidate the effects of Rapamycin on mTOR, S6 and Akt phosphorylation levels were analyzed by Western blot to gauge mTORC1 and mTORC2 activity (Figure 4(a)), respectively. We performed Western blots using the proteins isolated from mouse heart following $30 \mathrm{~min}$ of ischemia and 1 hour or 24 hours of reperfusion. S6 phosphorylation at Ser 235/236 was immensely increased following ischemia and $1 \mathrm{hr}$ as well as 24 hours of reperfusion as compared to Control (Figures 4(a) and 4(b)). The phosphorylation of S6 was significantly reduced with Rapamycin treatment $(n=5, p<0.0001)$ (Figures 4(a) and 4(b)). Total S6 was also induced following I/R as compared to Control, but it significantly reduced with Rapamycin treatment as compared to Control or I/R $(n=5, p<0.05)$ (Figures 4(a) and 4(c)). The phosphorylation of AKT $\left(\mathrm{Ser}^{473}\right.$ ) was reduced following ischemia and 24 hours of reperfusion 


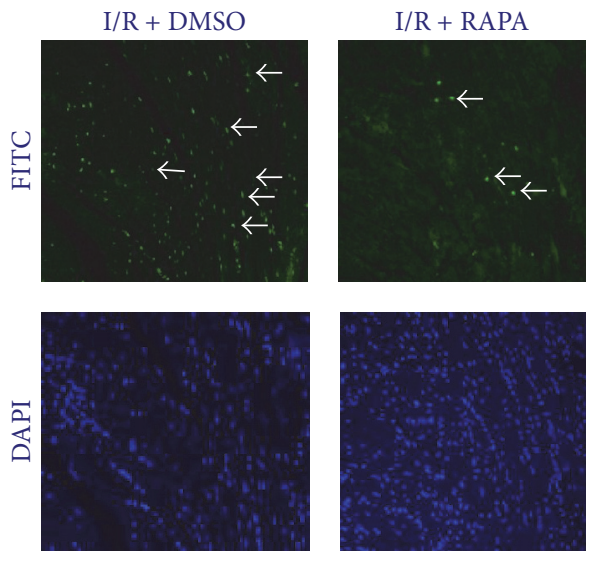

(a)

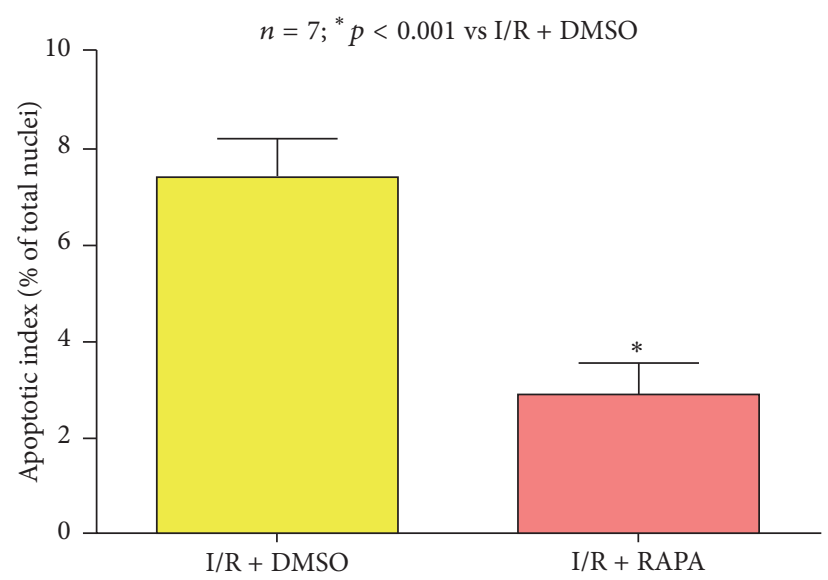

(b)

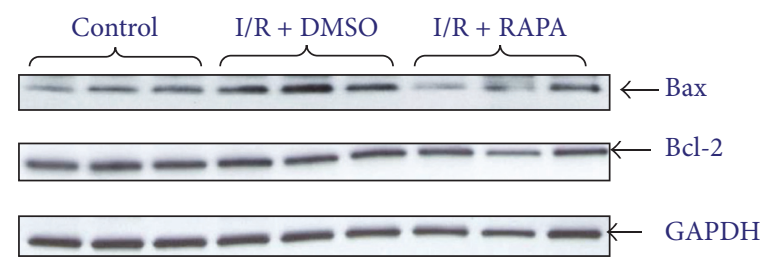

(c)

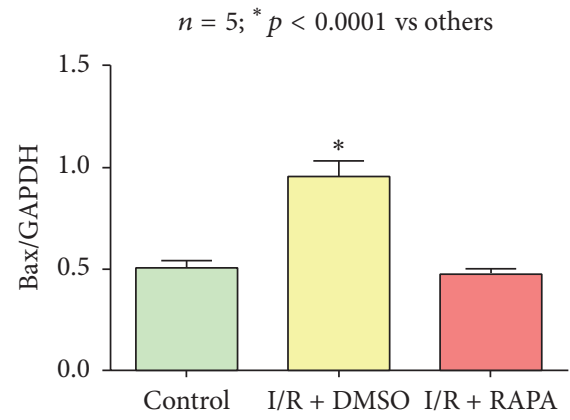

(d)

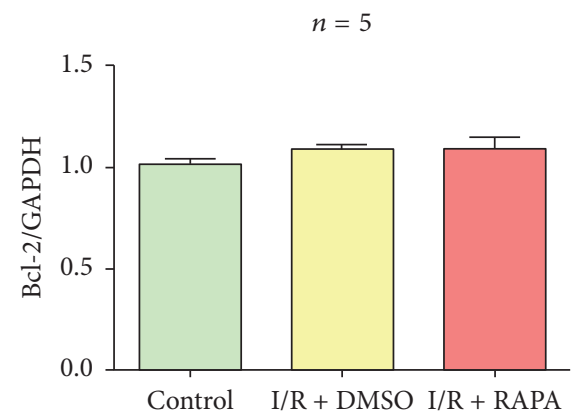

(e)

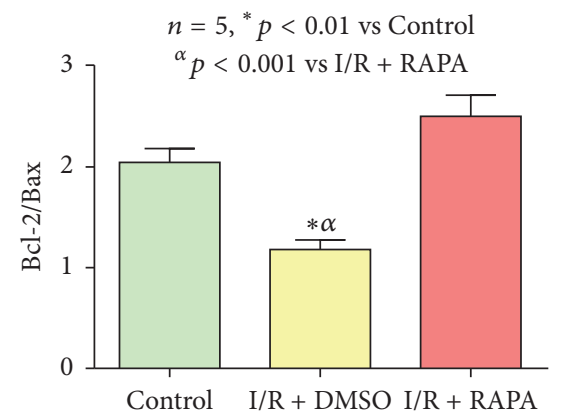

(f)

FIGURE 3: Effect of Rapamycin on myocardial apoptosis. (a) Representative images of TUNEL-positive nuclei in green fluorescent color and total nuclei staining with DAPI (blue) staining of sections of myocardium treated following 30 minutes of ischemia/24 hours of reperfusion. (b) Apoptotic index calculated as the \% of TUNEL stained apoptotic nuclei versus the total amount of nuclei visualized via DAPI staining. (c) Representative immunoblots of Bax and Bcl-2 in hearts of C57 mice following 30 minutes of ischemia and 24 hours of reperfusion including Control (C57), DMSO (I/R + DMSO), and Rapamycin (I/R + RAPA) treated groups ( $n=3 /$ group). GAPDH immunoblot was used as a loading Control. (d) Densitometric analysis of the ratio of Bax to GAPDH ( $n=5 /$ group). (e) Densitometric analysis of the ratio of Bcl-2 to GAPDH ( $n=5 /$ group). (f) Densitometric analysis of the ratio of Bcl- 2 to Bax ( $n=5 /$ group).

as compared to Control, although that reduction was not significant after 1 hour of reperfusion (Figures $4(\mathrm{a})$ and $4(\mathrm{~d})$ ). However, postischemic Rapamycin treatment was found to significantly increase phosphorylation at this site following 30 min ischemia and 24-hour reperfusion $(n=5, p<0.001)$ (Figures 4(a) and 4(d)). The induction of phosphorylation of AKT following 1 hour of reperfusion with Rapamycin was not significant. Total AKT levels were reduced following $30 \mathrm{~min}$ ischemia and 1-hour reperfusion $(n=5, p<0.001)$ but were not altered between groups following 24 hours of reperfusion (Figures 4(a) and 4(e)).

3.6. Effect of Rapamycin on MAP Kinase Signal Transduction. To analyze the effect of postischemic Rapamycin treatment on the MAP kinase signaling pathways following 1 hour or 24 hours of reperfusion, phosphorylation levels of ERK1/2 and P38 were measured via Western blot analysis (Figure 5(a)). p-ERK1/2 was not altered following 1 hour of reperfusion between groups. However, following 24 hours of reperfusion, p-ERK1/2 was significantly increased following postischemic treatment with Rapamycin as compared to the Control and DMSO-treated I/R groups $(n=5, p<0.001)$ (Figures 5(a) and 5(b)). Total ERK1/2 level significantly reduced following 1 hour of reperfusion $(n=5, p<0.001)$ but it was rescued by Rapamycin treatment. However, total ERK did not change among any of the treatment groups following 24 hours of reperfusion $(n=5, p>0.05)$ (Figures 5(a) and 5(c)). Phospho-p38 remained unchanged between Control and 


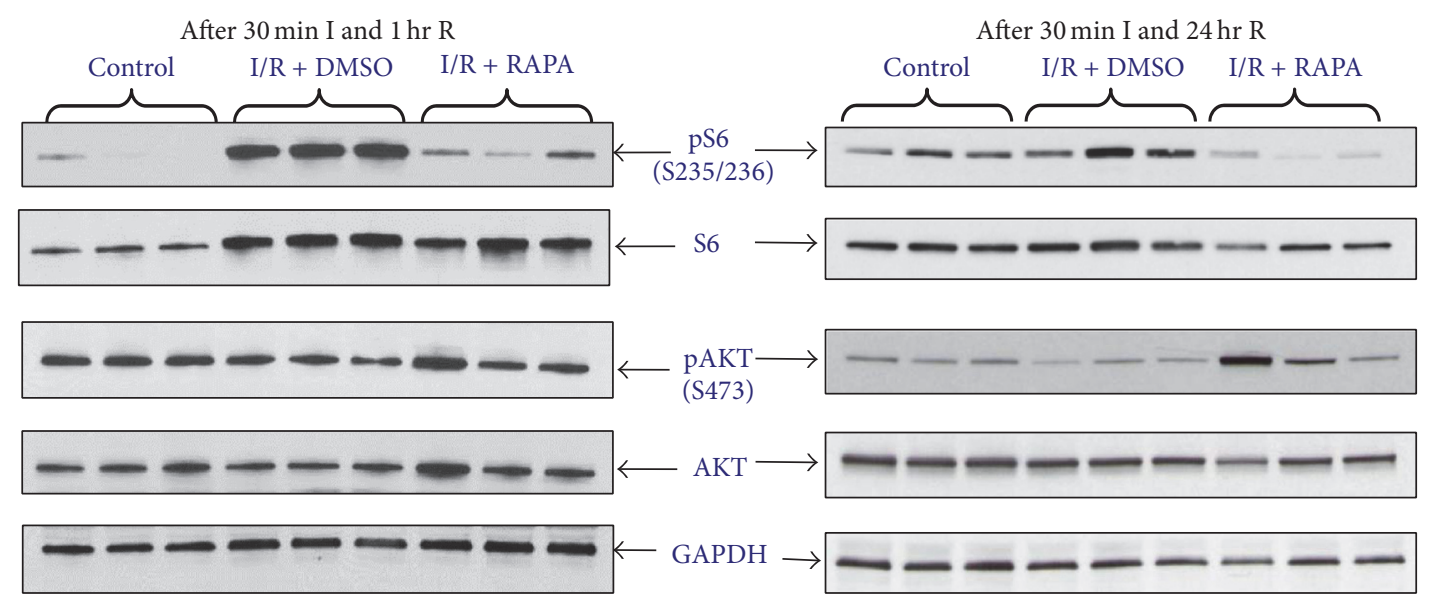

(a)

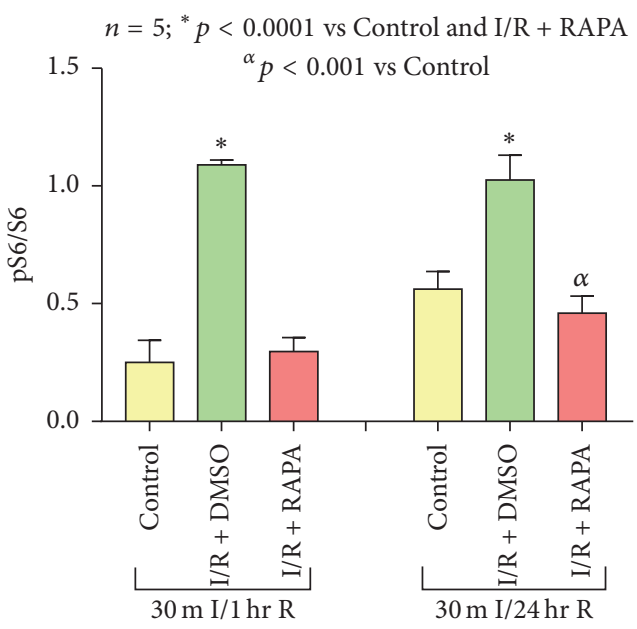

(b)

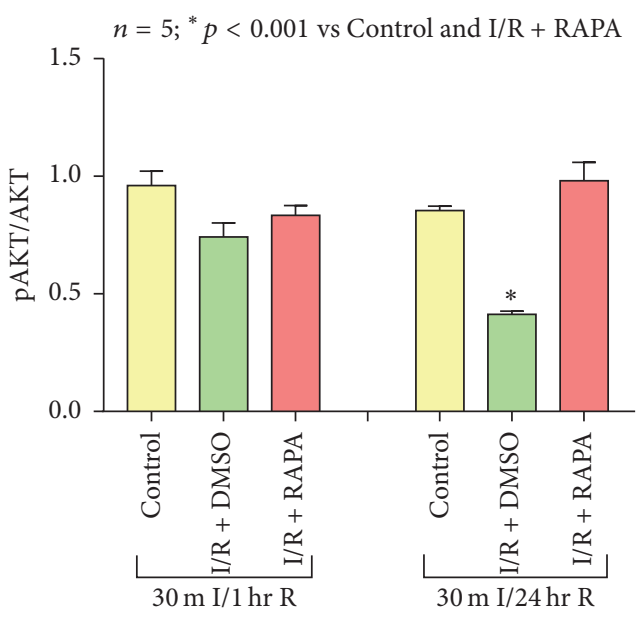

(d)

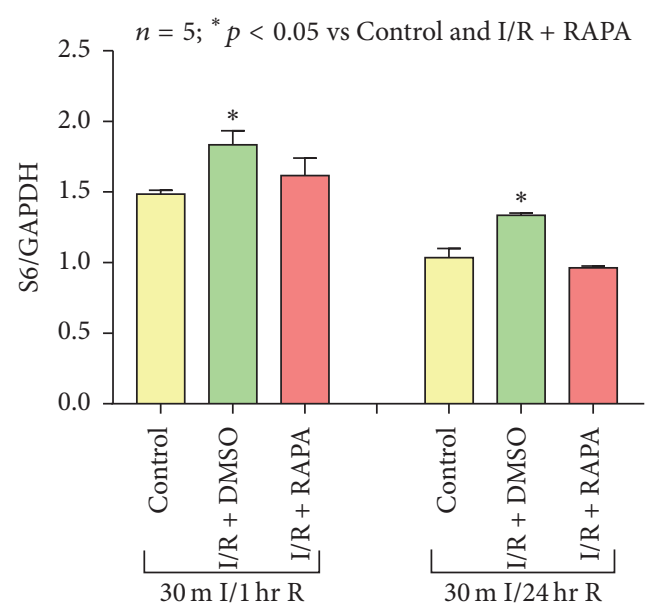

(c)

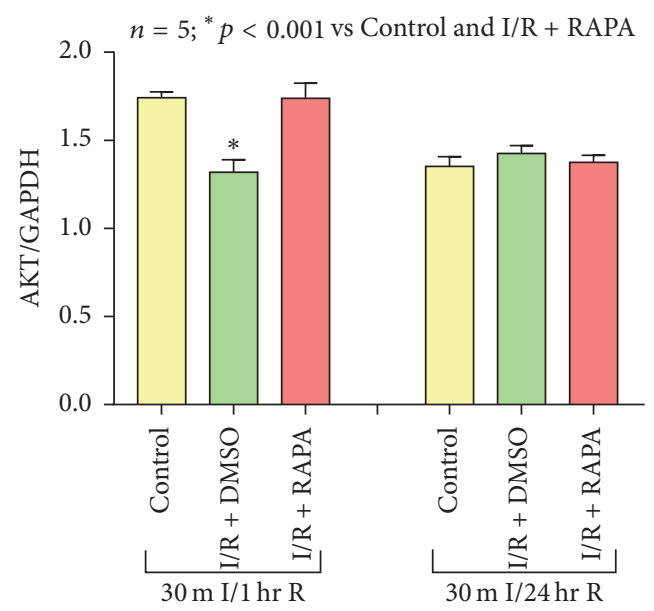

(e)

FIGURE 4: Rapamycin inhibits mTORC1 while simultaneously promoting mTORC2 activity. (a) Representative immunoblots of phosphorylated S6 (S235/236), total S6, phosphorylated AKT (S473), and total AKT in hearts of C57 mice following 30 minutes of ischemia and 1 hour and 24 hours of reperfusion including Control (C57), I/R + DMSO, and Rapamycin (I/R + RAPA) treated groups ( $n=3 /$ group). GAPDH immunoblots were used as a loading Control. (b) Densitometric analysis of the ratio of pS6 to S6. (c) Densitometric analysis of the ratio of S6 to GAPDH ( $n=5$ /group). (d) Densitometric analysis of the ratio of pAKT to AKT ( $n=5 /$ group). (e) Densitometric analysis of the ratio of AKT to GAPDH ( $n=5$ /group). 


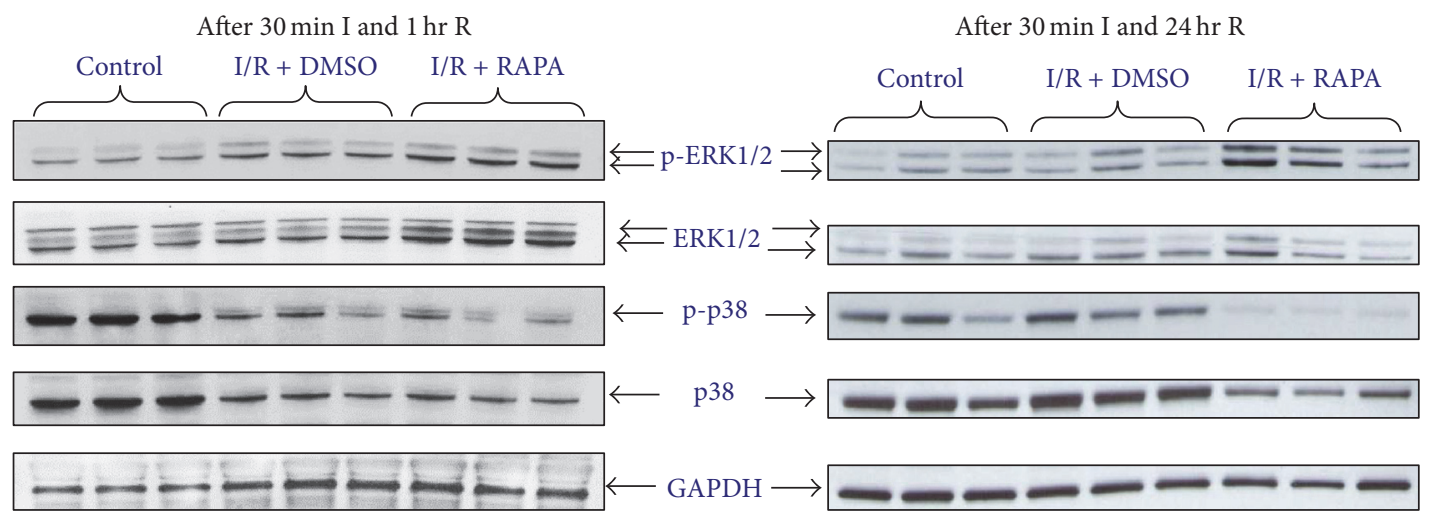

(a)

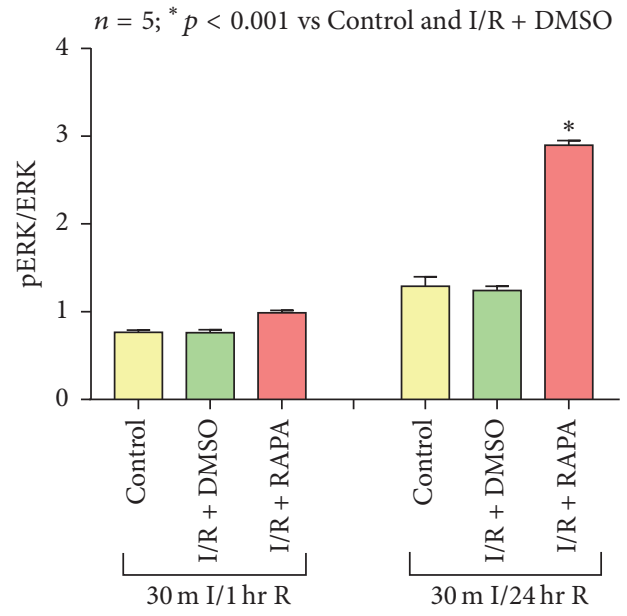

(b)

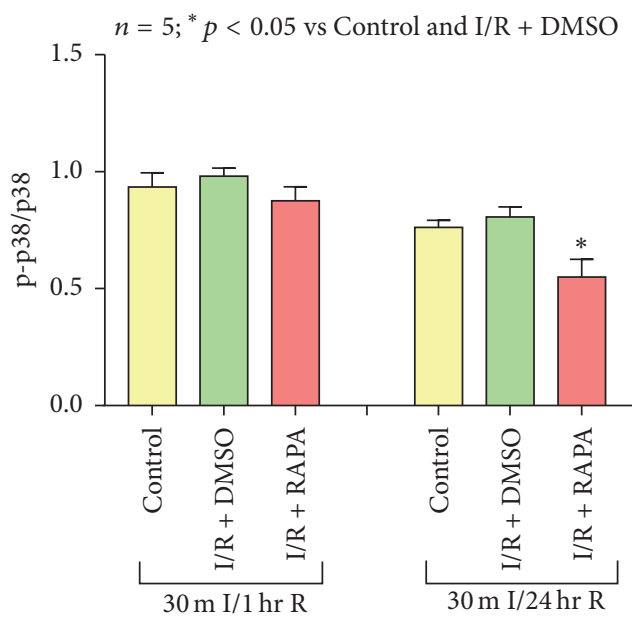

(d)

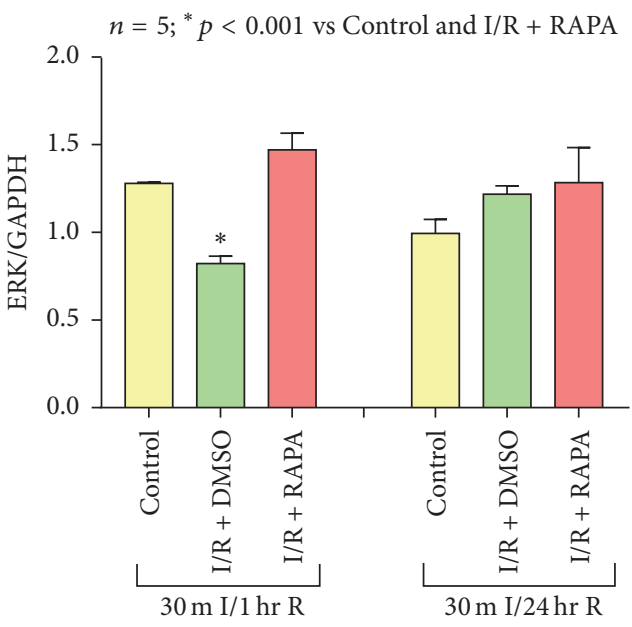

(c)

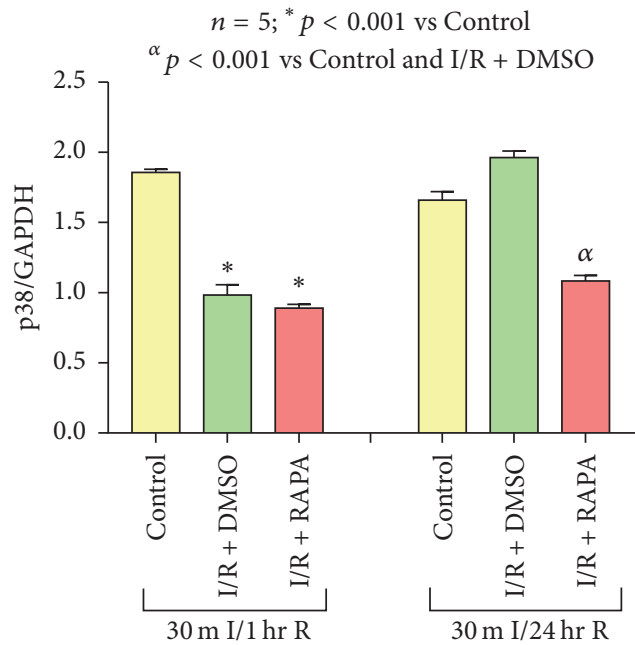

(e)

FIGURE 5: Effect of Rapamycin on MAP kinase signaling. (a) Representative immunoblots of phosphorylated ERK1/2, total ERK1/2, phosphorylated P38, and total P38 in hearts of C57 mice following 30 minutes of ischemia and 1 hour and 24 hours of reperfusion including Control (C57), DMSO (I/R + DMSO), and Rapamycin (I/R + RAPA) treated groups ( $n=3$ /group). GAPDH immunoblots were used as a loading Control. (b) Densitometric analysis of the ratio of pERK1/2 to ERK1/2 ( $n=5 /$ group). (c) Densitometric analysis of the ratio of ERK1/2 to GAPDH ( $n=5$ /group). (d) Densitometric analysis of the ratio of pP38 to P38. (e) Densitometric analysis of the ratio of P38 to GAPDH ( $n=5$ /group). 

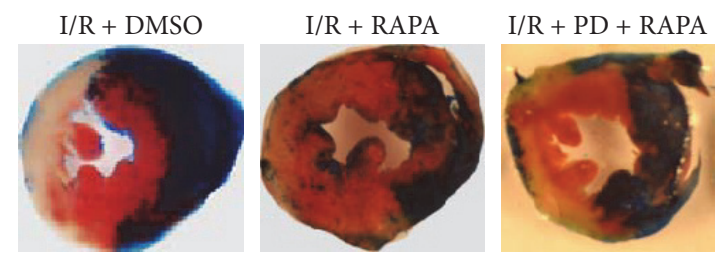

(a)

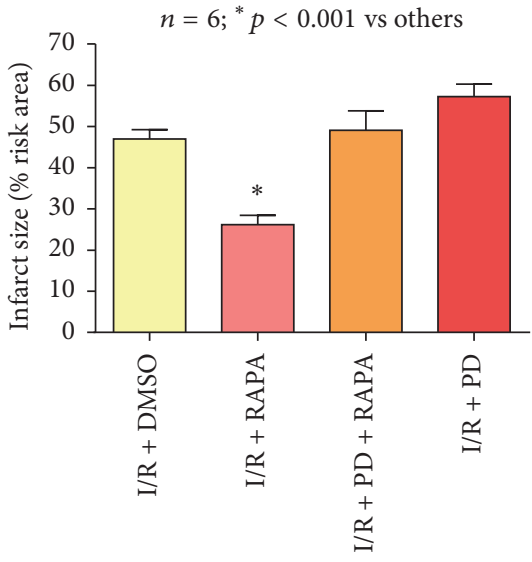

(b)

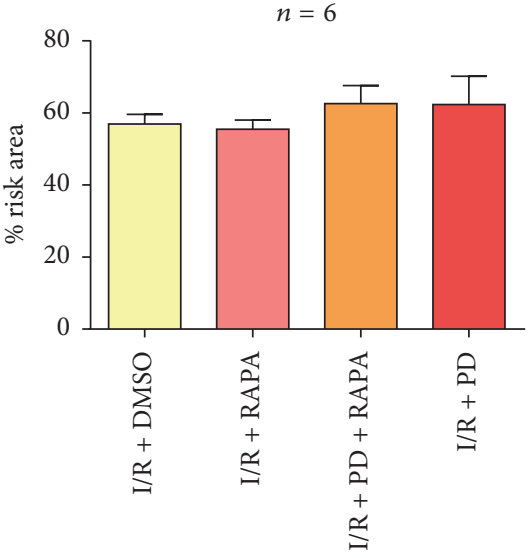

(c)

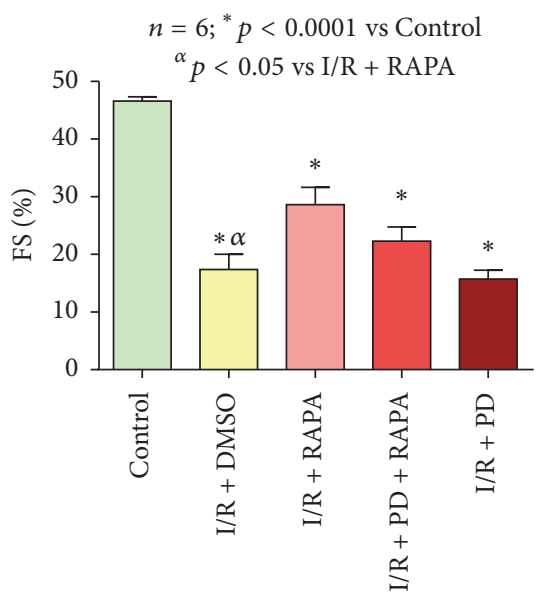

(d)

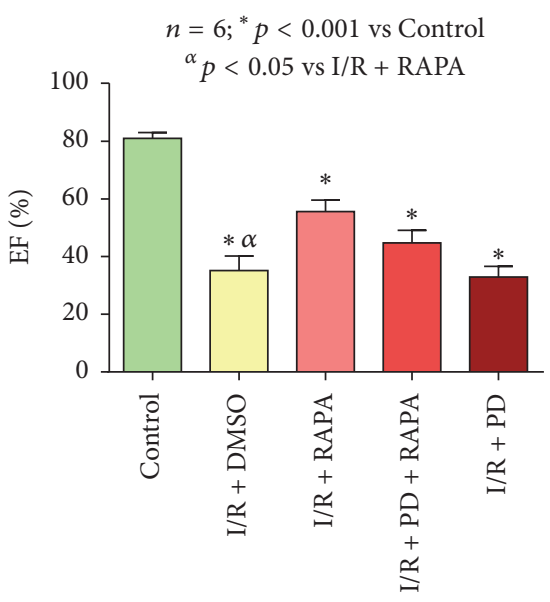

(e)

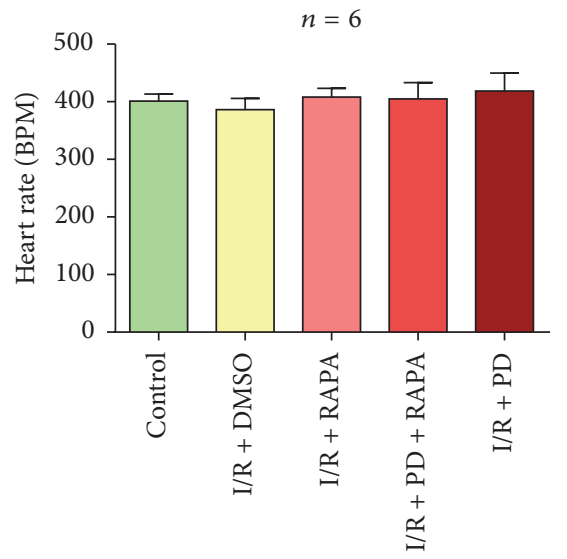

(f)

FIGURE 6: Effect of ERK inhibition on the infarct sparing effects of Rapamycin. (a) Whole tissue TTC and Phthalo blue staining of C57 mouse hearts following 30 minutes of ischemia and 24 hours of reperfusion with Rapamycin and/or PD98059. (b) Infarct size as a percentage of risk area. (c) Risk area as percent of LV. (d) Fractional shortening (\%) as measured by echocardiography. (e) Ejection fraction (\%). (f) Heart rate (BPM).

DMSO-treated I/R groups $(n=5, p>0.05)$ following 1 hour and 24 hours of reperfusion, but it significantly decreased with Rapamycin treatment following 24 hours of reperfusion $(n=5, p<0.01)$ (Figures 5(a), 5(d), and 5(e)).

\subsection{ERK Inhibition Abolishes the Infarct Sparing Effect of} Rapamycin. Rapamycin treatment significantly reduced infarct size (to $26.2 \pm 2.2 \%$ from $47.0 \pm 2.3 \%$ in the DMSOtreated Control group), which was completely blocked by treatment with ERK inhibitor, PD98059 (49.1 $\pm 4.7 \%, n=6$, $p<0.0001$ versus I/R + RAPA, Figure 6(b)). PD98059 alone did not alter the infarct size compared to DMSO-treated
$\mathrm{I} / \mathrm{R}$ group $(57.27 \pm 3.0 \%)$. Administration of PD98059 with/without Rapamycin did not alter total risk area $(62.65 \% \pm 1.8 \%$ and $62.36 \pm 3.3 \%)$ compared to Rapamycin$(55.52 \% \pm 2.5 \%)$ and DMSO- $(56.98 \% \pm 2.6 \%)$ treated I/R groups $(n=6, p>0.05)$ (Figure 6(c)).

Treatment with PD98059 also abolished post-I/R cardiac functional improvement induced by Rapamycin (FS, $22.31 \pm$ $2.5 \%$, and EF, $44.81 \pm 4.4 \%, n=6$, Figures $6(\mathrm{~d})$ and $6(\mathrm{e}))$. PostI/R cardiac function was not significantly different between PD98059 and DMSO-treated I/R mice (FS, $15.73 \pm 1.5 \%$, and $\mathrm{EF}, 32.95 \pm 3.6 \%)$. Heart rate did not change between the groups (Figure 6(f)). 
To investigate the effect of PD98059 on myocardial activation of ERK and AKT, we performed Western blot analysis using myocardial proteins from mice subjected to $30 \mathrm{~min}$ ischemia and 24-hour reperfusion and treated with PD98059 and Rapamycin. The results show that PD98059 inhibited Rapamycin-induced phosphorylation of ERK (Figures 7(a) and $7(d)$ ) but had no effect on the induction of phosphorylation of AKT by Rapamycin (Figures 7(a) and 7(b)).

\subsection{Rapamycin Attenuates Lipid Peroxidation through ERK} Activation. The oxidative stress as measured by accumulation of lipid peroxidation product malondialdehyde (MDA) was significantly increased in the hearts after 24-hour I/R injury as compared to nonischemic Control hearts $(n=4$; $p<0.0001$, Figure 8(a)). Rapamycin treatment at the onset of reperfusion significantly attenuated MDA accumulation following I/R ( $n=4, p<0.0001)$, suggesting a potent antioxidant-type activity of Rapamycin. Interestingly, ERK inhibitor PD98059 blocked the antioxidant effect of Rapamycin $(n=4, p<0.001)$. Postischemic treatment with Rapamycin also induced the expression of antioxidant enzymes (SOD-2, manganese-dependent superoxide dismutase, and ferritin heavy chain 1) in hearts as compared to Control and DMSO-treated I/R groups (Figures 8(b), 8(c), and 8(d)). Again, PD98059 abolished the induction of both antioxidant proteins with Rapamycin.

3.9. Effect of Rapamycin on STAT3 Signaling. Phosphorylation of STAT3 was significantly induced following I/R $(n=5$, $p<0.001$ after 1 hour of reperfusion and $p<0.01$ after 24 hours of reperfusion versus Control) (Figures 9(a) and 9(b)). Rapamycin treatment at reperfusion could not alter postischemic induction of phosphorylation of STAT3. Total STAT3 significantly reduced following ischemia and 1 hour of reperfusion ( $n=5, p<0.01$ versus control), but it was induced after 24 hours of reperfusion $(n=5 ; p<0.001$ versus Control) (Figures 9(a) and 9(c)). Rapamycin could not also alter the postischemic STAT3 levels following 1 hour as well as 24 hours of reperfusion.

\section{Discussion}

Early restoration of coronary flow is obligatory to resuscitate the ischemic myocardium. However, reperfusion may paradoxically contribute to myocardial dysfunction in the ischemic area via "reperfusion injury" [24-26]. Reperfusion injury results in cardiomyocyte damage through ventricular arrhythmias, myocardial stunning, microvascular obstruction, endothelial dysfunction, and irreversible cell damage or necrosis (termed lethal reperfusion injury) [27, 28]. As it stands, mitigation of reperfusion-induced cardiomyocyte death remains among the most attractive approaches to further improve outcomes following successful percutaneous coronary intervention in AMI patients. However, no pharmaceutical approaches are currently approved by the FDA that can directly protect heart against the deleterious effects of reperfusion injury due to the lack of a consistent clinical benefit.
In the present study, we investigated the potential therapeutic effect of Rapamycin when given at reperfusion in a mouse model of in vivo I/R. Our results show that Rapamycin $(0.25 \mathrm{mg} / \mathrm{kg}$, i.c. $)$ induced cardioprotection as shown by significant reduction of myocardial infarct size. In addition postI/R cardiomyocyte apoptosis was significantly diminished in the Rapamycin-treated group as compared to DMSO-treated mice as depicted by TUNEL staining. Echocardiography revealed that cardiac function was preserved in Rapamycintreated mice as compared to Control mice at 24 hours following I/R. Moreover, Rapamycin treatment inhibited the expression of proapoptotic protein Bax following I/R, without alteration of antiapoptotic protein $\mathrm{Bcl}-2$. The ratio of $\mathrm{Bcl}-$ 2/Bax expression was significantly increased in Rapamycintreated group suggesting a potent antiapoptotic effect in the myocardium following I/R.

Rapamycin selectively binds to FKBP12 and inhibits the kinase activity of mTORC1 [4, 6]. When bound, FKBP12 restricts access of mTOR substrate docking sites and destabilizes the structural integrity of mTORC1 [12, 29-31]. In acute doses, such as those utilized in the present study, mTORC2 is unaffected by this drug-receptor complex [18, 32]. mTOR, being a major growth regulator, plays an integral role in the induction of cardiac hypertrophy in response to pressure overload and MI [33]. Selective inhibition of MTORC1 with Rapamycin has been shown to preserve cardiac function and attenuate maladaptive hypertrophy resulting from these stressors [34-37].

Recent studies demonstrated that mTORC1 is the dominant complex stimulated by I/R injury, with mTORC 2 showing no significant changes in activity [38]. Genetic and pharmacologic evidence shows that mTORC1 inhibition is beneficial after myocardial infarction [39-41]; however, genetic deletion of mTORC2 is associated with increased ischemic damage [42]. PRAS40 overexpression reduced mortality and improved cardiac function after myocardial infarction via increased activity of mTORC2 signaling [42]. In the present study, we used the phosphorylation of S6 ( $\mathrm{Ser}^{235 / 236}$ ) and AKT $\left(\right.$ Ser $^{473}$ ) as measures for mTORC1 and mTORC2 activity, respectively. We examined the phosphorylation levels of S6 and AKT following 30 min of ischemia and 1 hour (early effect of reperfusion) as well as 24 hours of reperfusion (late effect of reperfusion).

Our results show that the phospho-S6 level increased following I/R, and the induction of phospho-S6 was abolished following treatment with postischemic Rapamycin therapy after 1 hour and 24 hours of reperfusion. Phospho-AKT levels showed no remarkable changes following $I / R$, but Rapamycin administration induced the phosphorylation of AKT after 24 hours of reperfusion. These results suggest that treatment with Rapamycin at reperfusion inhibits mTORC1, while simultaneously promoting mTORC2 signaling. This upregulation of phospho-AKT $\left(\mathrm{Ser}^{473}\right)$ in Rapamycin-treated mice at reperfusion may confer myocardial protection against I/R injury, which is comparable to that afforded by ischemic preconditioning [41].

The RISK pathway, representing a group of survival protein kinases that include the PI3K/AKT and ERK1/2, has been 


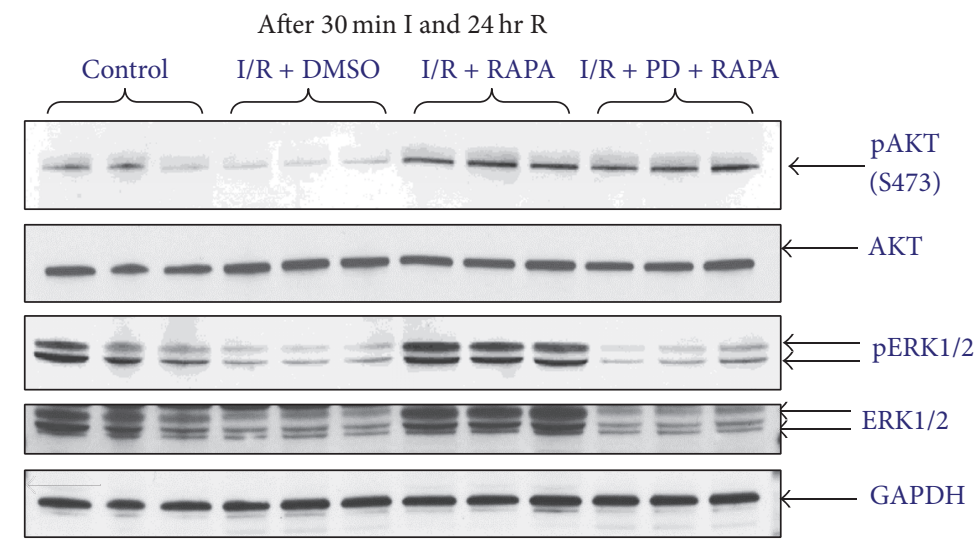

(a)

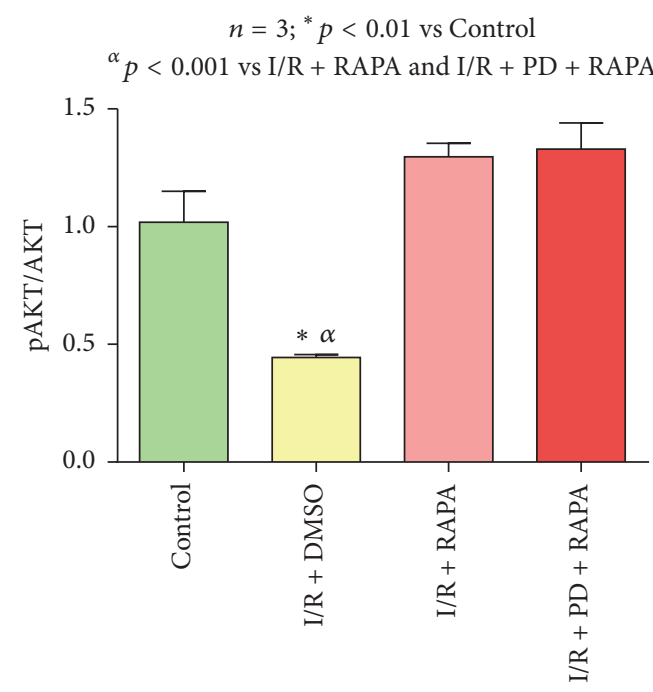

(b)

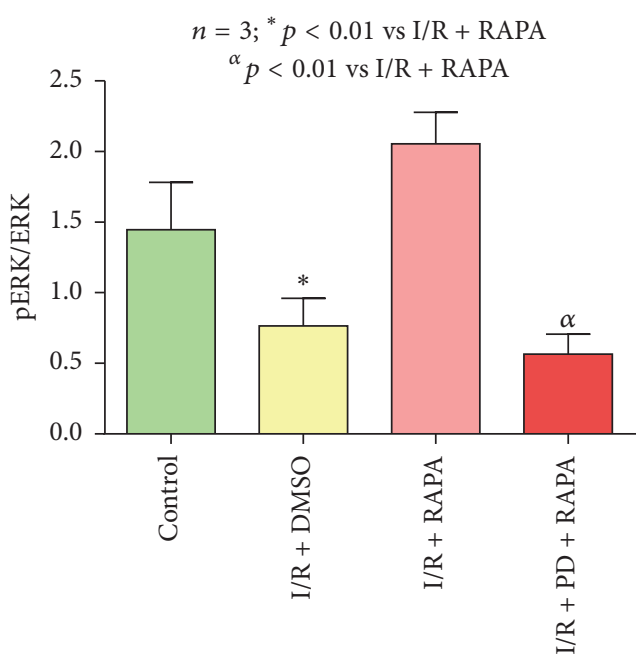

(d)

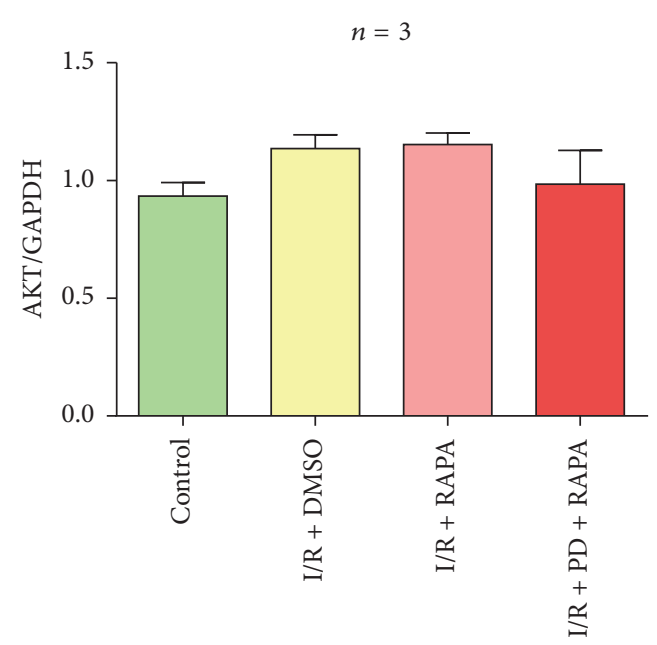

(c)

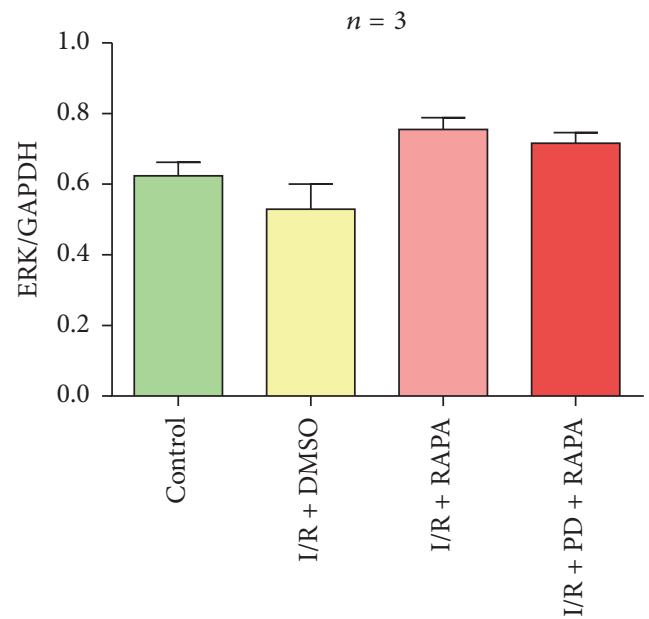

(e)

FIGURE 7: Effect of ERK inhibitor on phosphorylation of AKT and ERK. (a) Representative immunoblots of phosphorylated AKT, total AKT, phosphorylated ERK1/2, total ERK1/2, and GAPDH in hearts of C57 mice following 30 minutes of ischemia and 24 hours of reperfusion including Control (C57), DMSO (I/R + DMSO), Rapamycin (I/R + RAPA), and PD (I/R + PD + RAPA) treated groups ( $n=3 /$ group). (b) Densitometric analysis of the ratio of pAKT/AKT, (c) AKT/GAPDH, (d) pERK1/2 to ERK1/2, and (e) ERK1/2 to GAPDH. 


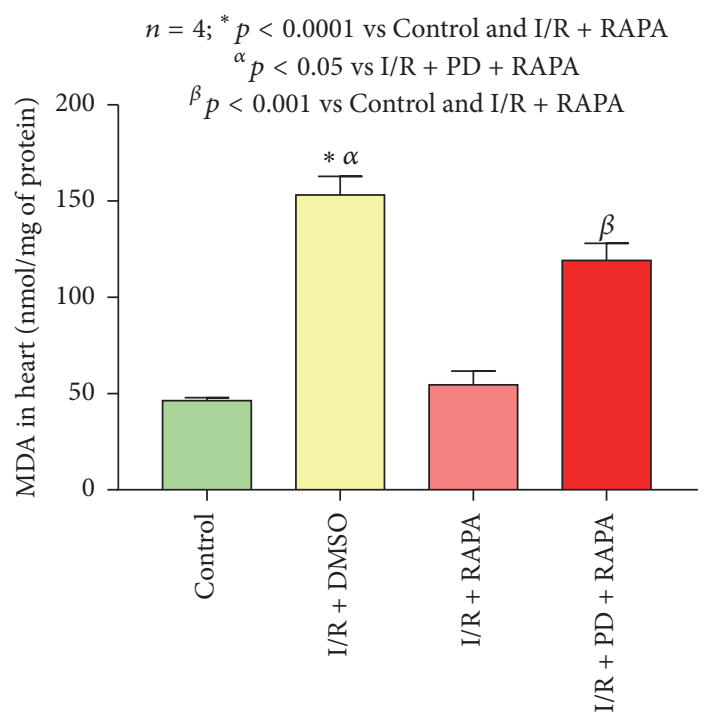

(a)

After $30 \mathrm{~min}$ I and $24 \mathrm{hr} \mathrm{R}$

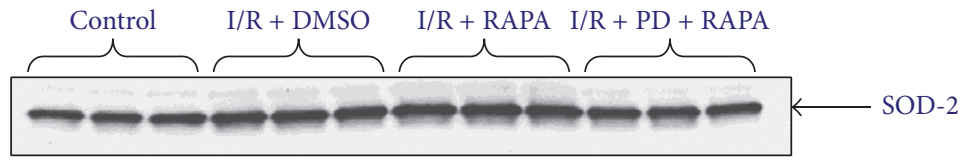

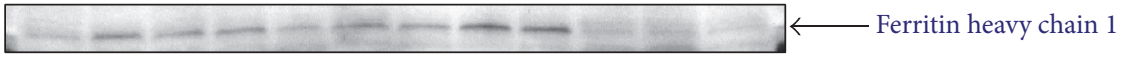

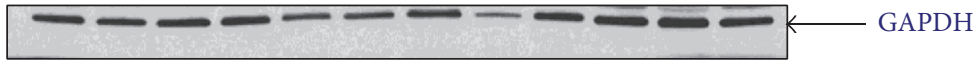

(b)

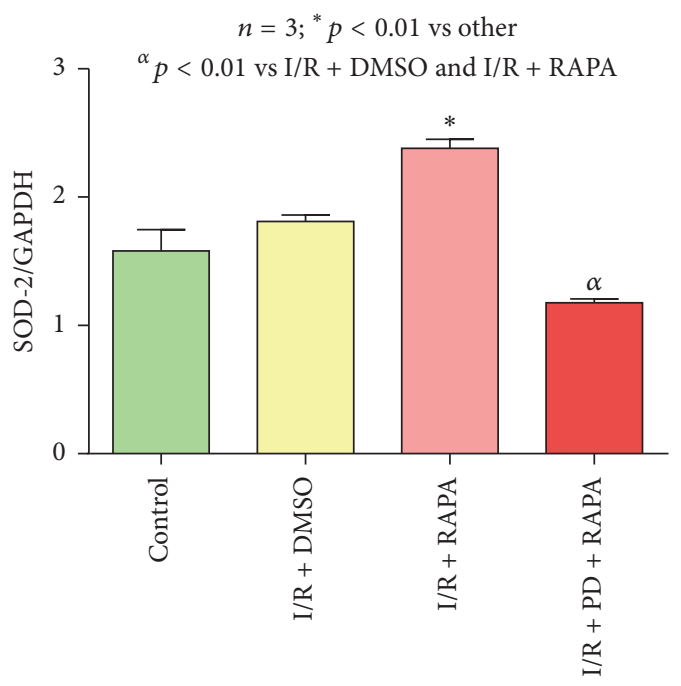

(c)

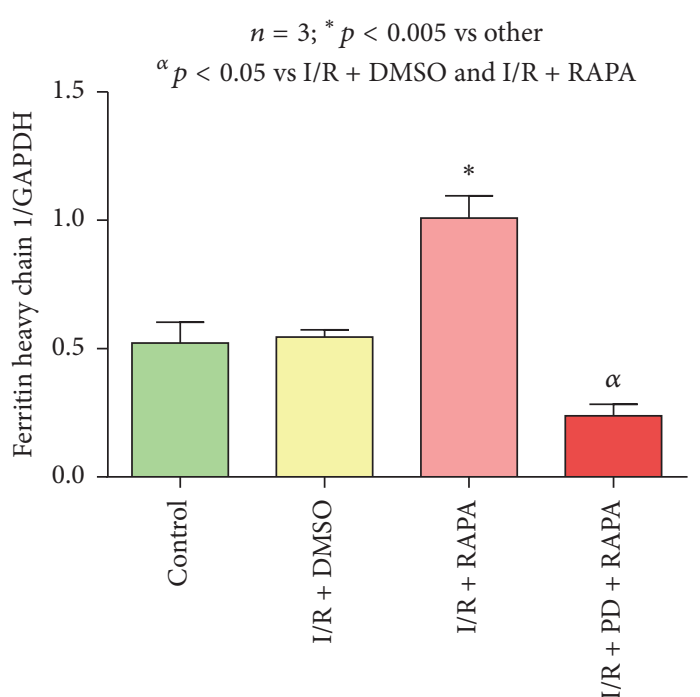

(d)

FIGURE 8: ERK inhibition abolishes the antioxidant effect of Rapamycin. (a) MDA level in hearts of C57 mice following 30 min ischemia and 24 hours of reperfusion with/without Rapamycin (RAPA) and/or PD98059 (RAPA + PD). (b) Representative immunoblots of SOD-2, ferritin heavy chain 1, and GAPDH in hearts of C57 mice following 30 minutes of ischemia and 24 hours of reperfusion including Control (C57), DMSO (I/R + DMSO), and Rapamycin (I/R + RAPA) and PD98059 (I/R + PD + RAPA) treated groups ( $n=3 /$ group). (c) Densitometric analysis of the ratio of SOD-2 to GAPDH ( $n=3$ /group). (d) Densitometric analysis of the ratio of ferritin heavy chain to GAPDH $(n=$ 3/group). 


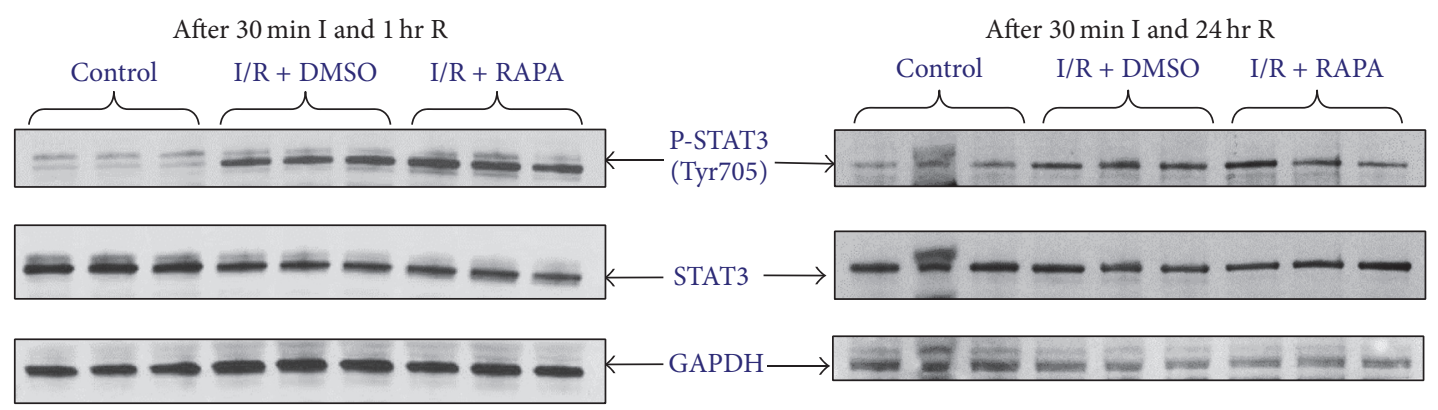

(a)

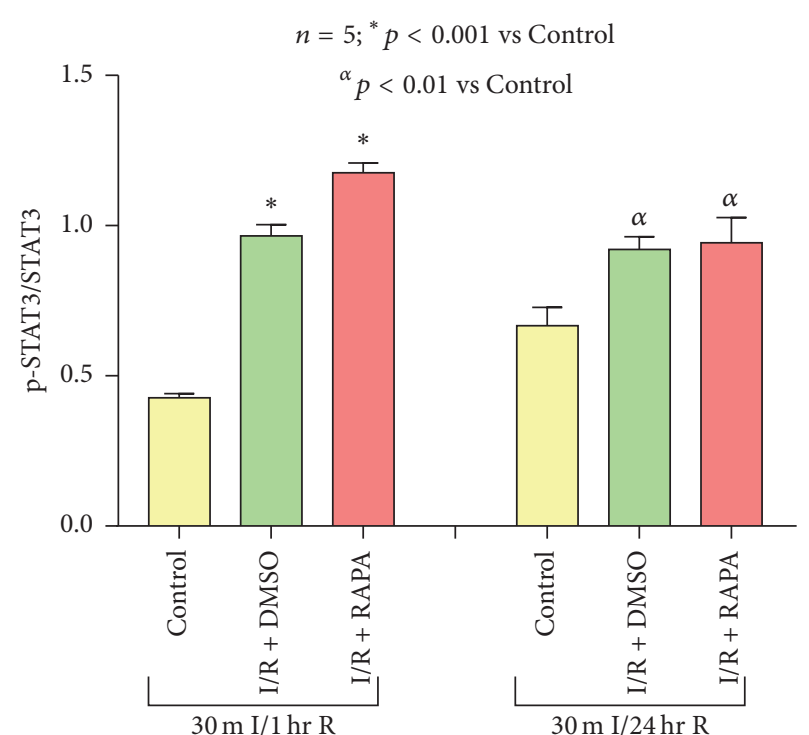

(b)

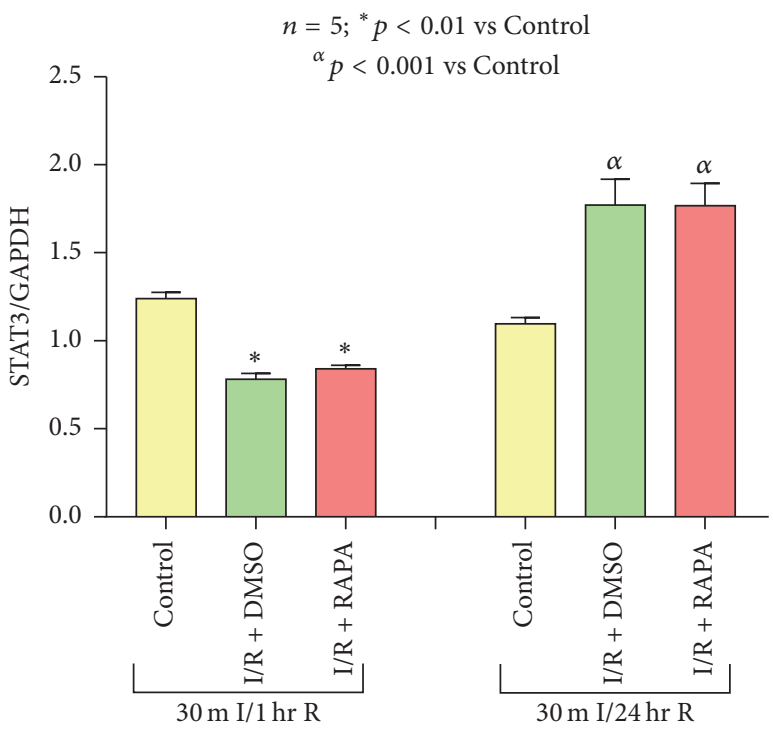

(c)

FIGURE 9: Effect of Rapamycin on STAT3 signaling. (a) Representative immunoblots of phosphorylated STAT3 and total STAT3 in hearts of C57 mice following 30 minutes of ischemia and 1 hour and 24 hours of reperfusion including Control (C57), DMSO (I/R + DMSO), and Rapamycin (I/R + RAPA) treated groups ( $n=3$ /group). GAPDH immunoblots were used as a loading Control. (b) Densitometric analysis of the ratio of pSTAT3 to STAT3 ( $n=5$ /group). (c) Densitometric analysis of the ratio of STAT3 to GAPDH ( $n=5 /$ group).

reported to exert cardioprotection when activated specifically at the time of myocardial reperfusion [20]. The cardioprotective effect of RISK pathway activation is associated with the recruitment of antiapoptotic signaling and the inhibition of proapoptotic proteins by avoiding the opening of the mitochondrial permeability transition pore at the onset of reperfusion [43-45]. Several studies have demonstrated that activating the RISK pathway with pharmacological agents may be a viable intervention for limiting myocardial infarction. Specifically, insulin, growth factors, TGB- $\beta 1$, leptin, apelin, opioids, adenosine, bradykinin, atorvastatin, metformin, guanylyl cyclase receptor atrial natriuretic peptide, and glucagon-like peptide elicit cardioprotection at the time of myocardial reperfusion through the activation of the RISK pathway [44]. In the present study, treatment with Rapamycin at the onset of reperfusion specifically activated AKT by inducing phosphorylation at the Ser 473 residue which may provide cardioprotective benefits.

The MAPK family of proteins encompass the second arm of the RISK signal pathway. While the apoptosis-inhibiting and infarct-reducing benefits of ERK1/2 upregulation have been well documented across multiple treatment models, there is still great debate regarding the role of p38 in cardioprotection [46-59]. In the present study, a significant increase in ERK1/2 phosphorylation was observed following Rapamycin administration at the onset of reperfusion and following 24 hours of reperfusion. Additionally, hearts subjected to $\mathrm{I} / \mathrm{R}$ without any pre- or posttreatment elicited no change in total ERK following 24 hours of reperfusion. These results are in line with previous studies published by our laboratory demonstrating the beneficial effects of preconditioning with Rapamycin as well as sildenafil (a phosphodiesterase inhibitor) [19,49]. p38 is particularly unique in the temporal aspects of its activation and inhibition. Despite being activated by myocardial I/R, previous evidence showed that preischemic inhibition of P38 MAPK reduced infarct size and preserved cardiac function in both in vivo and ex vivo mouse, rat, rabbit, and pig models [19, 49, 60-66]. A more recent study demonstrated that the benefits of p38 inhibition are time sensitive, with infarct-induced arrhythmias being attenuated only when p38 was inhibited before or during ischemia [67]. Conversely, ischemic preconditioning (IPC) 
has been shown to promote $\mathrm{p} 38$ phosphorylation at the time of myocardial reperfusion, indicating that modulation of these MAP kinases is subject to a complex array of signal transduction pathways over the entire course of ischemia and reperfusion [47, 50,52]. In the present study, phosphorylation of p38 was not altered after 1 hour of reperfusion between groups, but it has been inhibited following 24 hours of reperfusion with Rapamycin treatment. Total p38 was inhibited after ischemia and 1 hour of reperfusion, but it was induced following 24 hours of reperfusion. Rapamycin reduced the total p38 after 24 hours of reperfusion.

Inhibition of ERK via administration of the ERK inhibitor, PD98059, was performed in order to understand the cause and effect relationship between ERK activation and the reduction of infarct size following Rapamycin treatment at the end of ischemia. PD98059 completely abolished Rapamycin-induced infarct size reduction which was comparable with the DMSO group. Rapamycin-induced cardiac functional improvement following $\mathrm{I} / \mathrm{R}$ was also abolished following treatment with PD98059. These results suggest that ERK activation is a key factor in the cardioprotective benefits of Rapamycin therapy and is responsible for its overall infarct size reduction. PD98059 inhibited Rapamycin-induced ERK phosphorylation following 24 hours of reperfusion, which was not significantly different as compared to I/R group. However PD98059 had no effect on Rapamycin-induced AKT phosphorylation. These results suggest that Rapamycininduced AKT phosphorylation may have triggered ERK phosphorylation, because pharmacological inhibition of ERK had no effect on phosphorylation of AKT. Future studies are required to identify the precise sequence of activation of RISK signaling.

Many studies showed that reactive oxygen species (ROS) generated during early reperfusion lead to extensive oxidative stress to cells, which contributes to irreversible ischemic myocardial injury $[24,26,68]$. Earlier studies demonstrated the antioxidant effect of Rapamycin by protecting mitochondria against oxidative stress and apoptosis in a rat model of Parkinson disease [69], by improving endothelial function in aging [70] and vascular contractility [71]. Rapamycin also protects human corneal endothelial cells from oxidative injury-mediated cell death via inhibition of ROS production [72]. We previously reported the antioxidant effect of Rapamycin in reducing diabetes-induced myocardial dysfunction [7]. Rapamycin treatment reduced oxidative stress in diabetic heart by augmenting antioxidant proteins as well as iron-regulating proteins [7]. Ferritin heavy chain (FHC) protein plays an important role in the pathogenesis of heart failure [73]. A decrease in the abundance of ferritin heavy chain 1 enhances the levels of iron deposition and the resultant enhanced oxidative stress, which lead to cardiomyocytes cell death. In the present study, our results show that the lipid peroxidation product MDA was enhanced following $\mathrm{I} / \mathrm{R}$ in the heart, which was reduced by Rapamycin administration at the onset of reperfusion. The expressions of antioxidant proteins SOD-2 and iron-regulating protein ferritin heavy chain 1 were increased with Rapamycin treatment at the onset of reperfusion. Taken together, these data suggest that Rapamycin inhibits oxidative stress by inducing the expression of antioxidant enzyme as well as iron-regulating protein following $\mathrm{I} / \mathrm{R}$ injury.

In an effort to investigate the effects of Rapamycin as a clinical intervention for MI, we have collected strong evidence detailing the molecular signaling pathways by which the drug acts when administered at the onset of reperfusion. In addition to mTORC1 inhibition and mTORC2 induction, our data proves that Rapamycin also upregulates the ERK protein of the MAPK family. Recently we showed the beneficial effects of chronic treatment with Rapamycin in improving metabolic status and preventing cardiac dysfunction in diabetic mice ( $\mathrm{db} / \mathrm{db}$ mice) through attenuation of oxidative stress as well as alteration of contractile and glucose metabolic proteins $[6,7,13]$. Using diabetic mice, we demonstrated that reperfusion therapy with Rapamycin in diabetic hearts provides cardioprotection through the STAT3-AKT signaling pathway $[6,7,13]$. In the present study, we examined the effect of Rapamycin treatment at the onset of reperfusion on the phosphorylation of STAT3 in nondiabetic and normal mice. The phosphorylation of STAT3 was induced after ischemia and 1 hour as well as 24 hours of reperfusion. Total STAT3 reduced at an early time point of reperfusion but was induced after 24 hours of reperfusion. Rapamycin did not alter the effects of $I / R$ on phosphorylation as well as total level of STAT3 in the hearts of C57 mice. Therefore, the mechanism of cardioprotection against I/R injury by reperfusion therapy with Rapamycin is different in normal mice than in mice with diabetes.

\section{Conclusion}

The novel observation of this present study is that the reperfusion therapy with Rapamycin protects the heart against I/R injury via the AKT and ERK signaling pathway, but not through STAT3 signaling. Because sirolimus-eluting stents are safely used in patients with coronary artery disease, the infarct-reducing and antiapoptotic properties of the drug make it an excellent candidate for therapeutic intervention following an ischemic episode. Future preclinical studies of Rapamycin in higher organisms, including canines and nonhuman primates, would further enhance our understanding of the beneficial effect of reperfusion therapy with Rapamycin [74]. However, the broad-spectrum clinical use of mTOR inhibition is still awaiting a targeted pharmaceutical approach to specifically inhibit mTORC1 with concomitant activation of mTORC2 and the RISK pathway.

\section{Disclosure}

A part of the abstract in the manuscript was presented at the session of Myocardial Protection and Perioperative Management II at the American Heart Association Scientific Sessions in Orlando, Florida, 2015 (Circulation. 2015; 132:A13498).

\section{Competing Interests}

On behalf of all authors, the corresponding author states that there is no conflict of interests. 


\section{Authors' Contributions}

Scott M. Filippone and Arun Samidurai contributed equally to this study.

\section{Acknowledgments}

This work was supported by Grants from the National Institutes of Health RO1HL134366 (Anindita Das and Rakesh C. Kukreja), R01HL118808 (Rakesh C. Kukreja), and R01HL133167 (Fadi N. Salloum) and American Heart Association 14GRNT20010003 (Fadi N. Salloum), CTSA (UL1TR000058 from the National Center for Advancing Translational Sciences), the CCTR Endowment Fund of Virginia Commonwealth University (Anindita Das), and A. D. Williams Fund of Virginia Commonwealth University UL1RR031990 (Anindita Das).

\section{References}

[1] F. Meric-Bernstam and A. M. Gonzalez-Angulo, "Targeting the mTOR signaling network for cancer therapy," Journal of Clinical Oncology, vol. 27, no. 13, pp. 2278-2287, 2009.

[2] S. C. Johnson, P. S. Rabinovitch, and M. Kaeberlein, "MTOR is a key modulator of ageing and age-related disease," Nature, vol. 493, no. 7432, pp. 338-345, 2013.

[3] P. Kapahi, D. Chen, A. N. Rogers et al., "With TOR, less is more: a key role for the conserved nutrient-sensing TOR pathway in aging," Cell Metabolism, vol. 11, no. 6, pp. 453-465, 2010.

[4] M. Laplante and D. M. Sabatini, "MTOR signaling in growth control and disease," Cell, vol. 149, no. 2, pp. 274-293, 2012.

[5] S. Sciarretta, M. Volpe, and J. Sadoshima, "Mammalian target of rapamycin signaling in cardiac physiology and disease," Circulation Research, vol. 114, no. 3, pp. 549-564, 2014.

[6] S. Wullschleger, R. Loewith, and M. N. Hall, "TOR signaling in growth and metabolism," Cell, vol. 124, no. 3, pp. 471-484, 2006.

[7] A. Das, D. Durrant, S. Koka, F. N. Salloum, L. Xi, and R. C. Kukreja, "Mammalian target of rapamycin (mTOR) inhibition with rapamycin improves cardiac function in type 2 diabetic mice: potential role of attenuated oxidative stress and altered contractile protein expression," Journal of Biological Chemistry, vol. 289, no. 7, pp. 4145-4160, 2014.

[8] S. S. Deepa, M. E. Walsh, R. T. Hamilton et al., "Rapamycin modulates markers of mitochondrial biogenesis and fatty acid oxidation in the adipose tissue of $\mathrm{db} / \mathrm{db}$ mice," Journal of Biochemical and Pharmacological Research, vol. 1, pp. 114-123, 2013.

[9] S. A. Khan, F. Salloum, A. Das, L. Xi, G. W. Vetrovec, and R. C. Kukreja, "Rapamycin confers preconditioning-like protection against ischemia-reperfusion injury in isolated mouse heart and cardiomyocytes," Journal of Molecular and Cellular Cardiology, vol. 41, no. 2, pp. 256-264, 2006.

[10] D. W. Lamming, L. Ye, D. M. Sabatini, and J. A. Baur, "Rapalogs and mTOR inhibitors as anti-aging therapeutics," Journal of Clinical Investigation, vol. 123, no. 3, pp. 980-989, 2013.

[11] D. E. Martin and M. N. Hall, "The expanding TOR signaling network," Current Opinion in Cell Biology, vol. 17, no. 2, pp. 158166, 2005.

[12] H. Yang, D. G. Rudge, J. D. Koos, B. Vaidialingam, H. J. Yang, and N. P. Pavletich, "MTOR kinase structure, mechanism and regulation," Nature, vol. 497, no. 7448, pp. 217-223, 2013.
[13] A. Das, F. N. Salloum, S. M. Filippone et al., "Inhibition of mammalian target of rapamycin protects against reperfusion injury in diabetic heart through STAT3 signaling," Basic Research in Cardiology, vol. 110, no. 3, article no. 31, 2015.

[14] K. Hara, Y. Maruki, X. Long et al., "Raptor, a binding partner of target of rapamycin (TOR), mediates TOR action," Cell, vol. 110, no. 2, pp. 177-189, 2002.

[15] C. T. Keith and S. L. Schreiber, "PIK-related kinases: DNA repair, recombination, and cell cycle checkpoints," Science, vol. 270, no. 5233, pp. 50-51, 1995.

[16] T. Sato, A. Nakashima, L. Guo, and F. Tamanoi, "Specific activation of mTORC1 by Rheb G-protein in vitro involves enhanced recruitment of its substrate protein," The Journal of Biological Chemistry, vol. 284, no. 19, pp. 12783-12791, 2009.

[17] D. D. Sarbassov, D. A. Guertin, S. M. Ali, and D. M. Sabatini, "Phosphorylation and regulation of Akt/PKB by the rictormTOR complex," Science, vol. 307, no. 5712, pp. 1098-1101, 2005.

[18] D. D. Sarbassov, S. M. Ali, D.-H. Kim et al., "Rictor, a novel binding partner of mTOR, defines a rapamycin-insensitive and raptor-independent pathway that regulates the cytoskeleton," Current Biology, vol. 14, no. 14, pp. 1296-1302, 2004.

[19] A. Das, F. N. Salloum, D. Durrant, R. Ockaili, and R. C. Kukreja, "Rapamycin protects against myocardial ischemia-reperfusion injury through JAK2-STAT3 signaling pathway," Journal of Molecular and Cellular Cardiology, vol. 53, no. 6, pp. 858-869, 2012.

[20] D. J. Hausenloy and D. M. Yellon, "Reperfusion injury salvage kinase signalling: taking a RISK for cardioprotection," Heart Failure Reviews, vol. 12, no. 3-4, pp. 217-234, 2007.

[21] H. Kalakech, P. Hibert, D. Prunier-Mirebeau et al., "RISK and SAFE signaling pathway involvement in apolipoprotein A-Iinduced cardioprotection," PLOS ONE, vol. 9, no. 9, Article ID e107950, 2014.

[22] F. N. Salloum, A. Abbate, A. Das et al., "Sildenafil (Viagra) attenuates ischemic cardiomyopathy and improves left ventricular function in mice," American Journal of Physiology - Heart and Circulatory Physiology, vol. 294, no. 3, pp. H1398-H1406, 2008.

[23] J. W. Elrod, J. J. M. Greer, and D. J. Lefer, "Sildenafil-mediated acute cardioprotection is independent of the NO/cGMP pathway," American Journal of Physiology-Heart and Circulatory Physiology, vol. 292, no. 1, pp. H342-H347, 2007.

[24] G. Heusch, "Cardioprotection: chances and challenges of its translation to the clinic," The Lancet, vol. 381, no. 9861, pp. 166175, 2013.

[25] G. Heusch, "Molecular basis of cardioprotection: signal transduction in ischemic pre-, post-, and remote conditioning," Circulation Research, vol. 116, no. 4, pp. 674-699, 2015.

[26] D. M. Yellon and D. J. Hausenloy, "Myocardial reperfusion injury," The New England Journal of Medicine, vol. 357, no. 11, pp. 1121-1135, 2007.

[27] D. J. Hausenloy and D. M. Yellon, "Myocardial ischemiareperfusion injury: a neglected therapeutic target," Journal of Clinical Investigation, vol. 123, no. 1, pp. 92-100, 2013.

[28] S. Verma, P. W. M. Fedak, R. D. Weisel et al., "Fundamentals of reperfusion injury for the clinical cardiologist," Circulation, vol. 105, no. 20, pp. 2332-2336, 2002.

[29] F. Hausch, C. Kozany, M. Theodoropoulou, and A.-K. Fabian, "FKBPs and the Akt/mTOR pathway," Cell Cycle, vol. 12, no. 15, pp. 2366-2370, 2013.

[30] N. Oshiro, K.-I. Yoshino, S. Hidayat et al., "Dissociation of raptor from mTOR is a mechanism of rapamycin-induced 
inhibition of mTOR function," Genes to Cells, vol. 9, no. 4, pp. 359-366, 2004.

[31] C. K. Yip, K. Murata, T. Walz, D. M. Sabatini, and S. A. Kang, "Structure of the human mTOR complex i and its implications for rapamycin inhibition," Molecular Cell, vol.38, no. 5, pp. 768774, 2010.

[32] E. Jacinto, R. Loewith, A. Schmidt et al., "Mammalian TOR complex 2 controls the actin cytoskeleton and is rapamycin insensitive," Nature Cell Biology, vol. 6, no. 11, pp. 1122-1128, 2004.

[33] M. O. Boluyt, J.-S. Zheng, A. Younes et al., "Rapamycin inhibits $\alpha_{1}$-adrenergic receptor-stimulated cardiac myocyte hypertrophy but not activation of hypertrophy-associated genes: evidence for involvement of p70 S6 kinase," Circulation Research, vol. 81, no. 2, pp. 176-186, 1997.

[34] J. R. McMullen, M. C. Sherwood, O. Tarnavski et al., "Inhibition of mTOR signaling with rapamycin regresses established cardiac hypertrophy induced by pressure overload," Circulation, vol. 109, no. 24, pp. 3050-3055, 2004.

[35] T. Shioi, J. R. McMullen, O. Tarnavski et al., "Rapamycin attenuates load-induced cardiac hypertrophy in mice," Circulation, vol. 107, no. 12, pp. 1664-1670, 2003.

[36] M. Völkers, H. Toko, S. Doroudgar et al., "Pathological hypertrophy amelioration by PRAS40-mediated inhibition of mTORC1," Proceedings of the National Academy of Sciences of the United States of America, vol. 110, no. 31, pp. 12661-12666, 2013.

[37] X. Wu, Y. Cao, J. Nie et al., "Genetic and pharmacological inhibition of Rhebl-mTORC1 signaling exerts cardioprotection against adverse cardiac remodeling in mice," American Journal of Pathology, vol. 182, no. 6, pp. 2005-2014, 2013.

[38] T. Aoyagi, Y. Kusakari, C.-Y. Xiao et al., "Cardiac mTOR protects the heart against ischemia-reperfusion injury," American Journal of Physiology - Heart and Circulatory Physiology, vol. 303, no. 1, pp. H75-H85, 2012.

[39] S. J. Buss, S. Muenz, J. H. Riffel et al., "Beneficial Effects of Mammalian Target of Rapamycin Inhibition on Left Ventricular Remodeling After Myocardial Infarction," Journal of the American College of Cardiology, vol. 54, no. 25, pp. 2435-2446, 2009.

[40] S. Sciarretta, P. Zhai, D. Shao et al., "Rheb is a critical regulator of autophagy during myocardial ischemia: pathophysiological implications in obesity and metabolic syndrome," Circulation, vol. 125, no. 9, pp. 1134-1146, 2012.

[41] T. Yano, M. Ferlito, A. Aponte et al., "Pivotal role of mTORC2 and involvement of ribosomal protein $\mathrm{S} 6$ in cardioprotective signaling," Circulation Research, vol. 114, no. 8, pp. 1268-1280, 2014.

[42] M. Völkers, M. H. Konstandin, S. Doroudgar et al., "Mechanistic target of rapamycin complex 2 protects the heart from ischemic damage," Circulation, vol. 128, no. 19, pp. 2132-2144, 2013.

[43] S. R. Datta, H. Dudek, X. Tao et al., "Akt phosphorylation of BAD couples survival signals to the cell-intrinsic death machinery," Cell, vol. 91, no. 2, pp. 231-241, 1997.

[44] D. J. Hausenloy and D. M. Yellon, "New directions for protecting the heart against ischaemia-reperfusion injury: targeting the reperfusion injury salvage kinase (RISK)-pathway," Cardiovascular Research, vol. 61, no. 3, pp. 448-460, 2004.

[45] J. Zha, H. Harada, E. Yang, J. Jockel, and S. J. Korsmeyer, "Serine phosphorylation of death agonist BAD in response to survival factor results in binding to 14-3-3 not BCL-X(L)," Cell, vol. 87, no. 4, pp. 619-628, 1996.
[46] S. C. Armstrong, "Protein kinase activation and myocardial ischemia/reperfusion injury," Cardiovascular Research, vol. 61, no. 3, pp. 427-436, 2004.

[47] R. Da Silva, T. Grampp, T. Pasch, M. C. Schaub, and M. Zaugg, "Differential activation of mitogen-activated protein kinases in ischemic and anesthetic preconditioning," Anesthesiology, vol. 100, no. 1, pp. 59-69, 2004.

[48] A. Das, L. Xi, and R. C. Kukreja, "Protein kinase G-dependent cardioprotective mechanism of phosphodiesterase- 5 inhibition involves phosphorylation of ERK and GSK3 $\beta$," Journal of Biological Chemistry, vol. 283, no. 43, pp. 29572-29585, 2008.

[49] A. Das, F. N. Salloum, L. Xi, Y. J. Rao, and R. C. Kukreja, "ERK phosphorylation mediates sildenafil-induced myocardial protection against ischemia-reperfusion injury in mice," American Journal of Physiology - Heart and Circulatory Physiology, vol. 296, no. 5, pp. H1236-H1243, 2009.

[50] R. M. Fryer, H. H. Patel, A. K. Hsu, and G. J. Gross, "Stressactivated protein kinase phosphorylation during cardioprotection in the ischemic myocardium," American Journal of Physiology-Heart and Circulatory Physiology, vol. 281, no. 3, pp. H1184-H1192, 2001.

[51] R. M. Fryer, P. F. Pratt, A. K. Hsu, and G. J. Gross, "Differential activation of extracellular signal regulated kinase isoforms in preconditioning and opioid-induced cardioprotection," Journal of Pharmacology and Experimental Therapeutics, vol. 296, no. 2, pp. 642-649, 2001.

[52] D. J. Hausenloy and D. M. Yellon, "Survival kinases in ischemic preconditioning and postconditioning," Cardiovascular Research, vol. 70, no. 2, pp. 240-253, 2006.

[53] P. Ping and E. Murphy, "Role of p38 mitogen-activated protein kinases in preconditioning: a detrimental factor or a protective kinase?" Circulation Research, vol. 86, no. 9, pp. 921-922, 2000.

[54] N. Shimizu, M. Yoshiyama, T. Omura et al., "Activation of mitogen-activated protein kinases and activator protein-1 in myocardial infarction in rats," Cardiovascular Research, vol. 38, no. 1, pp. 116-124, 1998.

[55] C. Strohm, M. Barancik, M.-L. V. Brühl, S. A. R. Kilian, and W. Schaper, "Inhibition of the ER-kinase cascade by PD98059 and UO126 counteracts ischemic preconditioning in pig myocardium," Journal of Cardiovascular Pharmacology, vol. 36, no. 2, pp. 218-229, 2000.

[56] H.-Y. Sun, N.-P. Wang, M. Halkos et al., "Postconditioning attenuates cardiomyocyte apoptosis via inhibition of JNK and p38 mitogen-activated protein kinase signaling pathways," Apoptosis, vol. 11, no. 9, pp. 1583-1593, 2006.

[57] Z. Xu, X. Ji, and P. G. Boysen, "Exogenous nitric oxide generates ROS and induces cardioprotection: involvement of PKG, mitochondrial KATP channels, and ERK," American Journal of Physiology-Heart and Circulatory Physiology, vol. 286, no. 4, pp. H1433-H1440, 2004.

[58] X.-M. Yang, T. Krieg, L. Cui, J. M. Downey, and M. V. Cohen, "NECA and bradykinin at reperfusion reduce infarction in rabbit hearts by signaling through PI3K, ERK, and NO," Journal of Molecular and Cellular Cardiology, vol. 36, no. 3, pp. 411-421, 2004.

[59] T.-L. Yue, C. Wang, J.-L. Gu et al., "Inhibition of extracellular signal-regulated kinase enhances ischemia/reoxygenationinduced apoptosis in cultured cardiac myocytes and exaggerates reperfusion injury in isolated perfused heart," Circulation Research, vol. 86, no. 6, pp. 692-699, 2000.

[60] A. Aleshin, Y. Sawa, M. Ono, T. Funatsu, S. Miyagawa, and H. Matsuda, "Myocardial protective effect of FR167653; a novel 
cytokine inhibitor in ischemic-reperfused rat heart," European Journal of Cardio-Thoracic Surgery, vol. 26, no. 5, pp. 974-980, 2004.

[61] M. Barancik, P. Htun, C. Strohm, S. Kilian, and W. Schaper, "Inhibition of the cardiac p38-MAPK pathway by SB203580 delays ischemic cell death," Journal of Cardiovascular Pharmacology, vol. 35, no. 3, pp. 474-483, 2000.

[62] A. Gysembergh, B. Z. Simkhovich, R. A. Kloner, and K. Przyklenk, "p38 MAPK activity is not increased early during sustained coronary artery occlusion in preconditioned versus control rabbit heart," Journal of Molecular and Cellular Cardiology, vol. 33, no. 4, pp. 681-690, 2001.

[63] X. L. Ma, S. Kumar, F. Gao et al., "Inhibition of p38 mitogenactivated protein kinase decreases cardiomyocyte apoptosis and improves cardiac function after myocardial ischemia and reperfusion," Circulation, vol. 99, no. 13, pp. 1685-1691, 1999.

[64] K. Mackay and D. Mochly-Rosen, "An inhibitor of p38 mitogenactivated protein kinase protects neonatal cardiac myocytes from ischemia," The Journal of Biological Chemistry, vol. 274, no. 10, pp. 6272-6279, 1999.

[65] C. J. Thomas, D. C. H. Ng, N. Patsikatheodorou et al., "Cardioprotection from ischaemia-reperfusion injury by a novel flavonol that reduces activation of p38 MAPK," European Journal of Pharmacology, vol. 658, no. 2-3, pp. 160-167, 2011.

[66] M. Yada, A. Shimamoto, C. R. Hampton et al., "FR167653 diminishes infarct size in a murine model of myocardial ischemiareperfusion injury," Journal of Thoracic and Cardiovascular Surgery, vol. 128, no. 4, pp. 588-594, 2004.

[67] S. Surinkaew, S. Kumphune, S. Chattipakorn, and N. Chattipakorn, "Inhibition of p38 MAPK during ischemia, but not reperfusion, effectively attenuates fatal arrhythmia in ischemia/reperfusion heart," Journal of Cardiovascular Pharmacology, vol. 61, no. 2, pp. 133-141, 2013.

[68] K. Raedschelders, D. M. Ansley, and D. D. Y. Chen, “The cellular and molecular origin of reactive oxygen species generation during myocardial ischemia and reperfusion," Pharmacology and Therapeutics, vol. 133, no. 2, pp. 230-255, 2012.

[69] J. Jiang, J. Jiang, Y. Zuo, and Z. Gu, "Rapamycin protects the mitochondria against oxidative stress and apoptosis in a rat model of Parkinson's disease," International Journal of Molecular Medicine, vol. 31, no. 4, pp. 825-832, 2013.

[70] A. G. Rajapakse, G. Yepuri, J. M. Carvas et al., "Hyperactive S6K1 mediates oxidative stress and endothelial dysfunction in aging: inhibition by resveratrol," PLoS ONE, vol. 6, no. 4, Article ID e19237, 2011.

[71] G. Gao, J.-J. Li, Y. Li et al., "Rapamycin inhibits hydrogen peroxide-induced loss of vascular contractility," American Journal of Physiology - Heart and Circulatory Physiology, vol. 300, no. 5, pp. H1583-H1594, 2011.

[72] Y. J. Shin, D. Y. Cho, T. Y. Chung, S. B. Han, J. Y. Hyon, and W. R. Wee, "Rapamycin reduces reactive oxygen species in cultured human corneal endothelial cells," Current Eye Research, vol. 36, no. 12, pp. 1116-1122, 2011.

[73] S. Omiya, S. Hikoso, Y. Imanishi et al., "Downregulation of ferritin heavy chain increases labile iron pool, oxidative stress and cell death in cardiomyocytes," Journal of Molecular and Cellular Cardiology, vol. 46, no. 1, pp. 59-66, 2009.

[74] B. K. Kennedy and D. W. Lamming, "The mechanistic target of rapamycin: the grand conductor of metabolism and aging," Cell Metabolism, vol. 23, no. 6, pp. 990-1003, 2016. 


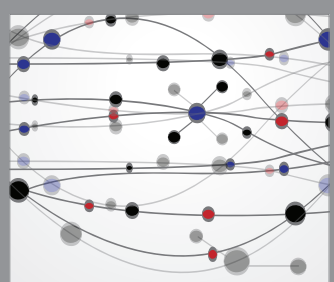

The Scientific World Journal
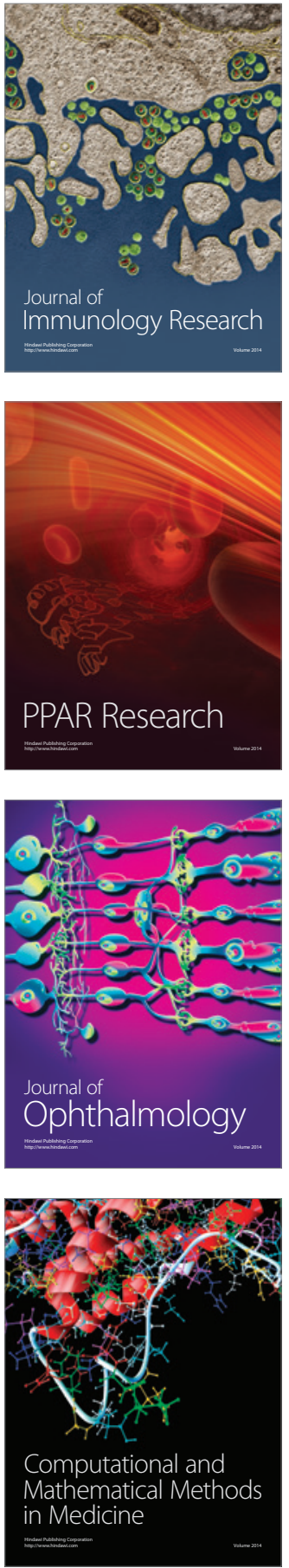

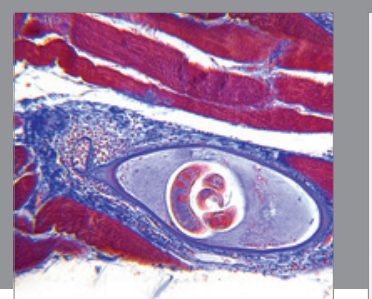

Gastroenterology Research and Practice
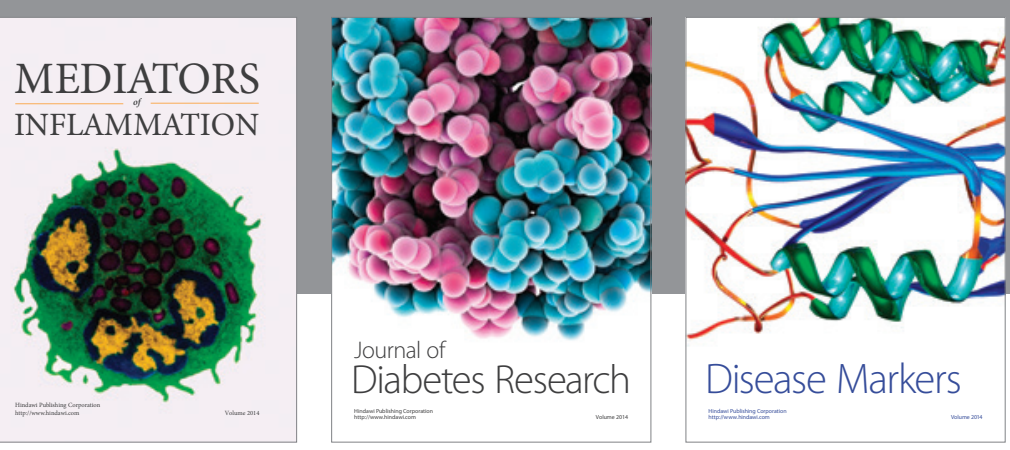

Disease Markers

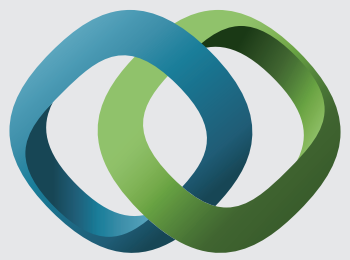

\section{Hindawi}

Submit your manuscripts at

https://www.hindawi.com
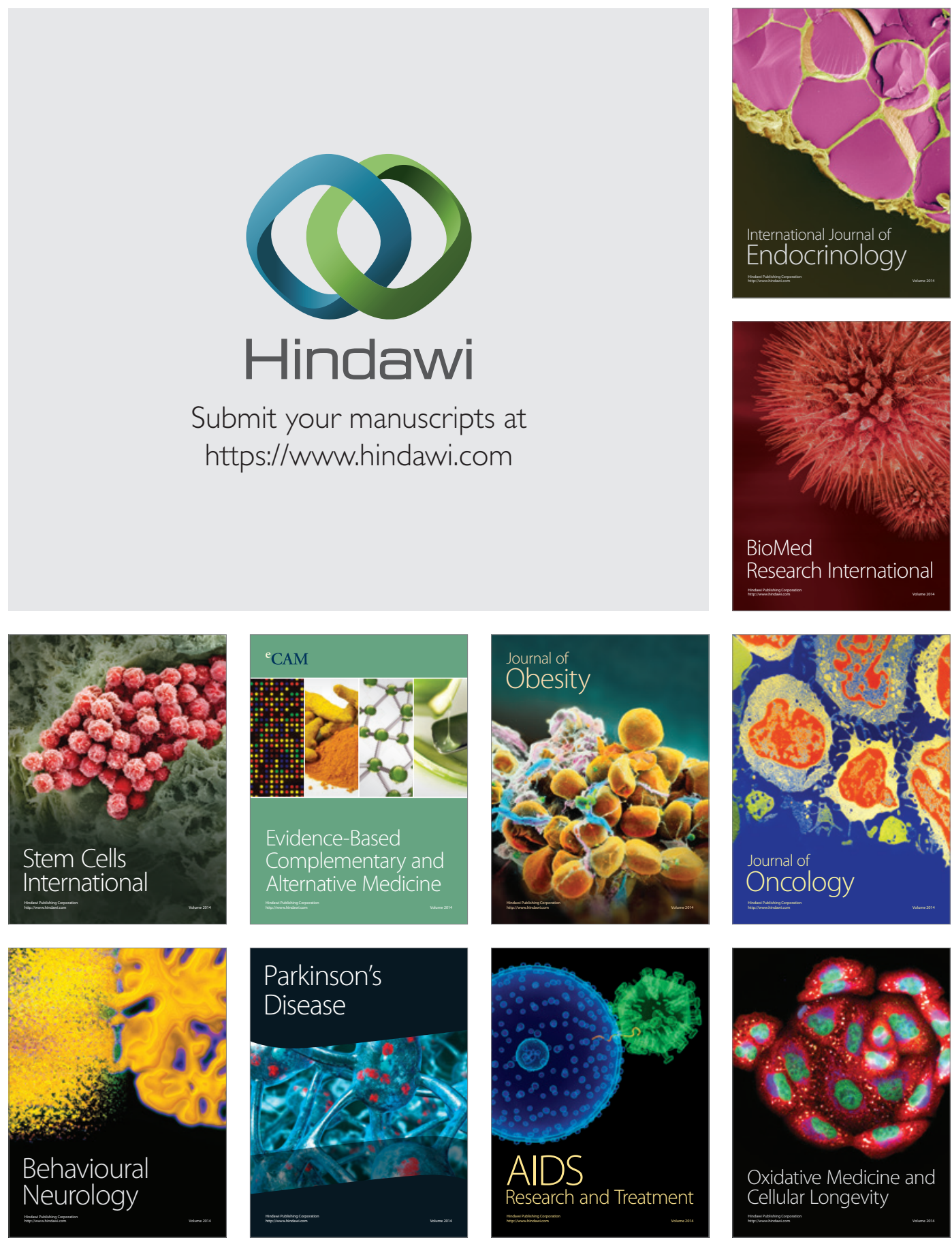